avances

en ciencias e

ingenierías

Artículo/Article Sección/Section A

\section{Spectral discretizations of the Darcy's equations with non standard boundary conditions}

\author{
J. M. Bernard ${ }^{*, * *}$ \\ *IUT d'Evry Val d'Essonne, 22, allee Jean-Rostand, 91025 Evry Cedex, France. \\ **Laboratoire d'analyse et probabilite d'Evry Val d'Essonne, Boulevard F. Mitterand, 91025 Evry Cedex, \\ France.
}

\section{Abstract}

This paper is devoted to the approximation of a nonstandard Darcy problem, which modelizes the ow in porous media, by spectral methods: the pressure is assigned on a part of the boundary. We propose two variational formulations, as well as three spectral discretizations. The second discretization improves the approximation of the divergence-free condition, but the error estimate on the pressure is not optimal, while the third one leads to optimal error estimate with a divergence-free discrete solution, which is important for some applications. Next, their numerical analysis is performed in detail and we present some numerical experiments which conrm the interest of the third discretization.

Keywords: Key words: Spectral methods; Darcy equations; boundary conditions; numerical results. 


\section{Introduction}

We consider the following Darcy problem:

$$
\begin{array}{lr}
\mathbf{u}+\nabla p=\mathbf{f} & \text { in } \Omega, \\
\operatorname{div} \mathbf{u}=0 & \text { in } \Omega, \\
\mathbf{u} \cdot \mathbf{n}=U_{0} & \text { on } \Gamma^{1}, \\
p=\varphi & \text { on } \Gamma^{2},
\end{array}
$$

where $\Omega$ is the plane square $]-1,1\left[2\right.$ and $\mathbf{n}=\left(n_{1}, n_{2}\right)$ is the exterior unit normal to the boundary $\Gamma=\partial \Omega$. The boundary $\Gamma$ is divided into two parts: the horizontal portion $\Gamma^{1}=$ $\{(x, y) \mid-1<x<1, y= \pm 1\}$ and the vertical portion $\Gamma^{2}=\{(x, y) \mid x= \pm 1,-1<y<1\}$. As we can see, the boundary condition on $\Gamma^{2}$ are nonstandard, since we prescribe the value of the pressure on $\Gamma^{2}$. On the contrary, we have a classical condition on the portion $\Gamma^{1}$. These conditions are described in the following figure.

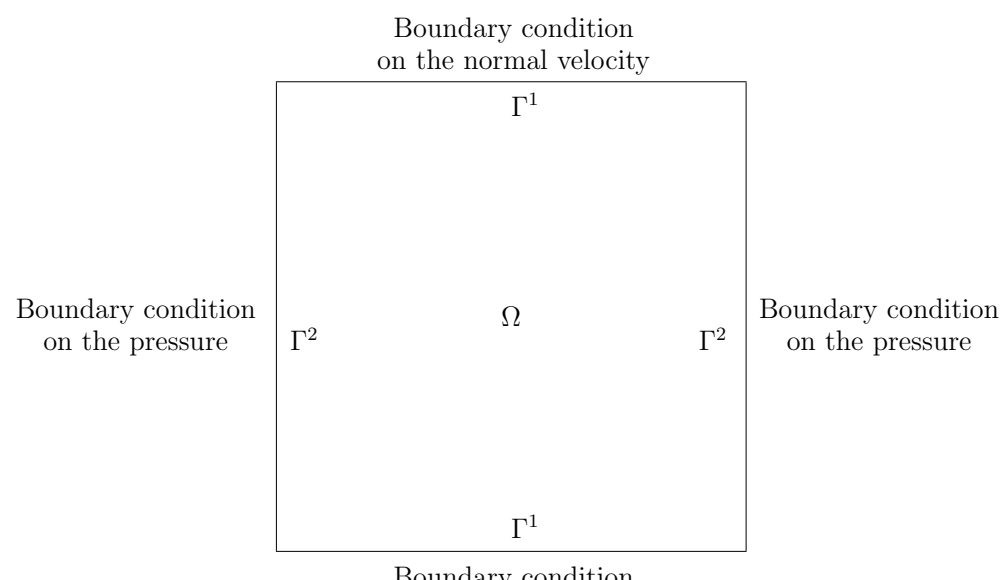

$$
\begin{aligned}
& \text { Boundary condition } \\
& \text { on the normal velocity }
\end{aligned}
$$

Figure 1.1

The equations of the Darcy problem not only modelize the flow in porous media, but also appear in the projection techniques for the solution of Navier-Stokes equations (see $[10]$ and [15]). The nonstandard boundary conditions, where the pressure is assigned on a part of the boundary, have a physical meaning: typically the portion $\Gamma^{1}$ corresponds to rigid walls, whereas the entry or exit of the fluids takes place through $\Gamma^{2}$. The spectral discretization with this type of boundary conditions was only studied within the framework of the Stokes problem (see [6], [4] and [5]), while Azaïez, Bernardi and Grundmann proposed in [2] the spectral discretization of the standard Darcy problem, where the normal velocity is assigned on the boundary.

This paper is devoted to the spectral discretization of the nonstandard Darcy problem. First, we give two variational formulations. Each one leads us to well-posed problems. Second, we study the regularity of the solution by using a mixed problem of DirichletNeumann for the Laplace operator. Next, from the first variational formulation, we derive a first spectral discretization, which is simple, but, in order to improve the approximation of the divergence-free condition, we study a second spectral discretization, where the inf-sup condition is obtained with more difficulty and where the error estimate on the pressure is not optimal. Finally, the second variational formulation yields a third spectral discretization, which leads us to optimal error estimate and a divergence-free discrete solution. 
An outline of this paper is as follows. The two continuous variational problems, as well as the regularity of the solution, are studied in Section 2. Section 3 is devoted to the analysis of three spectral approximations of this problem in the case of homogeneous boundary conditions. In Section 4, we present the algorithms that are used to solve the first and third discretizations, together with some numerical experiments.

\section{Statement of the problem and notation}

In order to set this problem into adequate spaces, recall the definition of the following standard Sobolev spaces (cf. J. Nečas [13]). For any multi-index $k=\left(k_{1}, k_{2}\right)$ with $k_{i} \geq 0$, set $|k|=k_{1}+k_{2}$ and denote

$$
\partial^{k} v=\frac{\partial^{|k|} v}{\partial x_{1}^{k_{1}} \partial x_{2}^{k_{2}}}
$$

Then for any integer $m \geq 0$ and any plane domain $\Omega$ whose boundary is Lipschitzcontinuous(cf. Grisvard [12]), we define:

$$
H^{m}(\Omega)=\left\{v \in L^{2}(\Omega) ; \partial^{k} v \in L^{2}(\Omega) \text { for } 1 \leq|k| \leq m\right\},
$$

equipped with the seminorm

$$
|v|_{H^{m}(\Omega)}=\left(\sum_{|k|=m} \int_{\Omega}\left|\partial^{k} v\right|^{2} d \mathbf{x}\right)^{\frac{1}{2}},
$$

and norm(for which it is an Hilbert space)

$$
\|v\|_{H^{m}(\Omega)}=\left(\sum_{|k|=0}^{m} \sum_{k}\left\|\partial^{k} v\right\|_{L^{2}(\Omega)}^{2}\right)^{1 / 2} .
$$

For extensions of this definition to non-integral values of $m$ (see [11,12]), let $s$ a real number such that $s=m+\sigma$ with $m \in \mathbb{N}$ and $0<\sigma<1$. We denote by $H^{s}(\Omega)$ the space of all distributions $u$ defined in $\Omega$ such that $u \in H^{m}(\Omega)$ and, $\forall|\alpha|=m$,

$$
\int_{\Omega} \int_{\Omega} \frac{\left(\partial^{\alpha} u(x)-\partial^{\alpha} u(y)\right)^{2}}{\|x-y\|^{2+2 \sigma}} d x d y<+\infty .
$$

It can be shown that $H^{s}(\Omega)$ is a Hilbert space for the scalar product

$$
(u, v)_{s, \Omega}=(u, v)_{m, \Omega}+\sum_{|\alpha|=m} \int_{\Omega} \int_{\Omega} \frac{\left(\partial^{\alpha} u(x)-\partial^{\alpha} u(y)\right)\left(\partial^{\alpha} v(x)-\partial^{\alpha} v(y)\right)}{\|x-y\|^{2+2 \sigma}} d x d y .
$$

Let $\Gamma^{\prime}$ be an open part of the boundary $\partial \Omega$ of class $C^{m-1,1}$ and $T_{1}^{\Gamma^{\prime}}$ the mapping $v \mapsto v_{\mid \Gamma^{\prime}}$ defined on $H^{m}(\Omega)$. We denote by $H^{m-\frac{1}{2}}\left(\Gamma^{\prime}\right)($ see $[7,12])$ the space $T_{1}^{\Gamma^{\prime}}\left(H^{m}(\Omega)\right)$ which is equipped with the norm:

$$
\|\varphi\|_{H^{m-\frac{1}{2}}}\left(\Gamma^{\prime}\right)=\inf \left\{\|v\|_{H^{m}(\Omega)}, v \in H^{m}(\Omega) \text { and } v_{\mid \Gamma^{\prime}}=\varphi\right\} .
$$

In this text, we shall use the spaces $H^{1 / 2}\left(\Gamma^{\prime}\right)$ and $H^{3 / 2}\left(\Gamma^{\prime}\right)$ corresponding to $m=1$ and 2 .

Let us define the space $H_{00}^{1 / 2}\left(\Gamma^{\prime}\right)=\left\{v_{\mid \Gamma^{\prime}}, v \in H^{1}(\Omega), \forall \mathbf{x} \in \partial \Omega \backslash \Gamma^{\prime}, v_{\mid \partial \Omega}(\mathbf{x})=0\right\}$. We shall also be interested in the dual space of $H_{00}^{1 / 2}\left(\Gamma^{\prime}\right)$,

$$
H^{-1 / 2}\left(\Gamma^{\prime}\right)=\left(H_{00}^{1 / 2}\left(\Gamma^{\prime}\right)\right)^{\prime} .
$$

We shall use the Hilbert space $H(\operatorname{div} ; \Omega)=\left\{\mathbf{v} \in L^{2}(\Omega)^{2}: \operatorname{div} \mathbf{v} \in L^{2}(\Omega)\right\}$, equipped with the norm 


$$
\|\mathbf{v}\|_{H(\operatorname{div} ; \Omega)}=\left(\left(\|\mathbf{v}\|_{L^{2}(\Omega)}\right)^{2}+\left(\|\operatorname{div} \mathbf{v}\|_{L^{2}(\Omega)}\right)^{2}\right)^{\frac{1}{2}} .
$$

For vanishing boundary values, we define:

$$
H_{0}^{1}(\Omega)=\left\{v \in H^{1}(\Omega): v_{\mid \partial \Omega}=0\right\} .
$$

For $\Lambda=]-1,1\left[\right.$, we denote the norm in $L^{2}(\Lambda)$ by $\|v\|_{0, \Lambda}=\left(\int_{-1}^{1}(v(x))^{2} d x\right)^{\frac{1}{2}}$, the seminorm in $H^{1}(\Lambda)$ by $|v|_{1, \Lambda}=\left(\int_{-1}^{1}\left(v^{\prime}(x)\right)^{2} d x\right)^{\frac{1}{2}}$ and the norm in $H^{1}(\Omega)$ by $\|v\|_{1, \Lambda}=\left(\int_{-1}^{1}\left((v(x))^{2}+\left(v^{\prime}(x)\right)^{2}\right) d x\right)^{\frac{1}{2}}$.

We note $\mathbf{x}=(x, y)$ the generic point of the square $\Omega$ and we call $\Gamma_{I}, \Gamma_{I I}, \Gamma_{I I I}$ and $\Gamma_{I V}$ the edges of $\Omega$, starting from west and turning counterclockwise. For each $J$, $J=I, I I, I I I, I V$, the extremities of the edge $\Gamma_{J}$ are $\mathbf{a}_{\mathbf{J}-\mathbf{1}}$ and $\mathbf{a}_{\mathbf{J}}$, with the convention $\mathbf{a}_{\mathbf{0}}=\mathbf{a}_{\mathbf{I V}}$, the exterior unit normal vector to $\Gamma_{J}$ is denoted by $\mathbf{n}_{\mathbf{J}}$ and the counterclockwise unit tangent vector is $\boldsymbol{\tau}_{\mathbf{J}}$. Figure 1.2 below presents this notation.

For any domain $\Delta$ in $\mathbb{R}$ or $\mathbb{R}^{2}$ and for any nonnegative integer $n, \mathbb{P}_{n}(\Delta)$ stands for the space of all polynomials on $\Delta$ with degree $\leq n$ with respect to each variable. We also use the notation $\mathbb{P}_{n}^{0}(\Delta)$ for the subspace $\mathbb{P}_{n}(\Delta) \cap H_{0}^{1}(\Delta)$. For $\left.\Lambda=\right]-1,1[$, the family $\left(L_{n}\right)_{n}$ of Legendre polynomials is a basis of the spaces $\mathbb{P}(\Lambda)$ of polynomials on $\Lambda$ (we refer to [7, Chap. I] for the properties of the orthogonal polynomials). These polynomials are orthogonal to each other in $L^{2}(\Lambda)$ and are caracterized as follows: for any integer $n \geq 0$, the polynomial $L_{n}$ is of degree $n$ and satisfies $L_{n}(1)=1$. Let us recall some properties that we need. The family $\left(L_{n}\right)_{n}$ is given by the recursion relation:

$$
\left\{\begin{array}{l}
L_{0}=1, L_{1}(\zeta)=\zeta \\
(n+1) L_{n+1}(\zeta)=(2 n+1) \zeta L_{n}(\zeta)-n L_{n-1}(\zeta), \quad n \geq 1 .
\end{array}\right.
$$

Each polynomial is a solution of the differential equation

$$
\left(\left(1-\zeta^{2}\right) L_{n}^{\prime}\right)^{\prime}+n(n+1) L_{n}=0, \quad n \geq 0,
$$

and its norm is given by

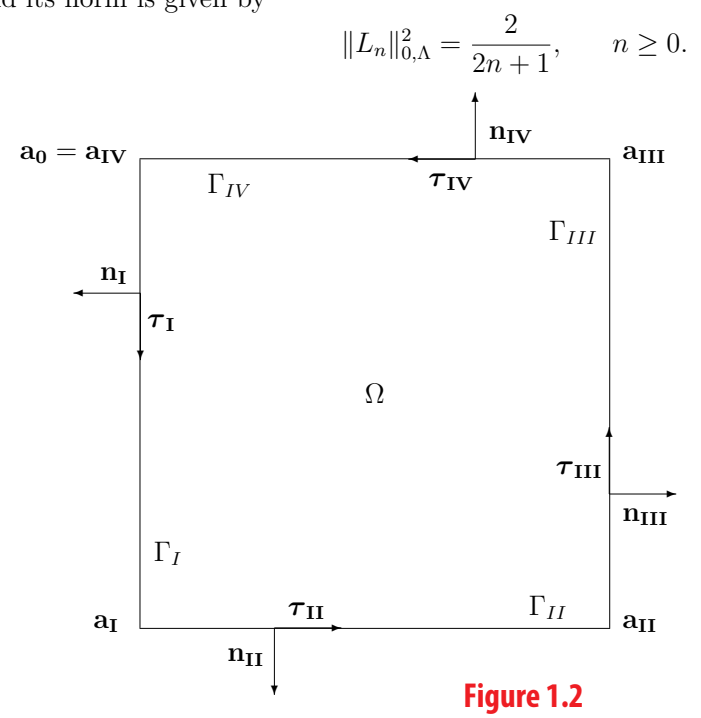

Three consecutive polynomials are linked by the integral equation 


$$
\int_{-1}^{\zeta} L_{n}(\xi) d \xi=\frac{1}{2 n+1}\left(L_{n+1}(\zeta)-L_{n-1}(\zeta)\right), \quad n \geq 1 .
$$

From (2.6) and integration by parts, we derive

$$
\begin{aligned}
L_{n}^{\prime}(1) & =\frac{n(n+1)}{2},\left\|L_{n}^{\prime}\right\|_{0, \Lambda}^{2}=n(n+1), \\
\forall \varphi_{n} & \in \mathbb{P}_{n}(\Lambda),\left|\varphi_{n}\right|_{1, \Lambda} \leq \sqrt{3} n^{2}\left\|\varphi_{n}\right\|_{0, \Lambda} .
\end{aligned}
$$

Next, let $N \geq 2$ be a fixed integer. We denote by $\xi_{j}, 0 \leq j \leq N$, the zeros of the polynomial $\left(1-\zeta^{2}\right) L_{N}^{\prime}(\zeta)$ in increasing order. We recall (see [7, Chap. I]) that there exist positive weights $\rho_{j}, 0 \leq j \leq N$, such that the following equality, called the Gauss-Lobatto formula, holds

$$
\forall \Phi \in \mathbb{P}_{2 N-1}(\Lambda), \int_{-1}^{1} \Phi(\zeta) d \zeta=\sum_{j=0}^{N} \Phi\left(\xi_{j}\right) \rho_{j} .
$$

Moreover, it follows from the identity (see [7, Chap. III])

$$
\sum_{j=0}^{N} L_{N}\left(\xi_{j}\right)^{2} \rho_{j}=\left(2+\frac{1}{N}\right)\left\|L_{N}\right\|_{0, \Lambda}^{2},
$$

that the bilinear form: $(u, v) \rightarrow \sum_{j=1}^{N} u\left(\xi_{j}\right) v\left(\xi_{j}\right) \rho_{j}$ is a scalar product on $\mathbb{P}_{N}(\Lambda)$, since we have

$$
\forall v \in \mathbb{P}_{N}(\Lambda),\|v\|_{0, \Lambda}^{2} \leq \sum_{j=0}^{N} v\left(\xi_{j}\right)^{2} \rho_{j} \leq 3\|v\|_{0, \Lambda}^{2} .
$$

\section{THE CONTINUOUS PROBLEM}

\section{First variational formulation}

We define the subspace $H^{1}\left(\Omega ; \Gamma^{\prime}\right)=\left\{v \in H^{1}(\Omega) \mid v=0\right.$ on $\left.\Gamma^{\prime}\right\}$ of $H^{1}(\Omega)$ and we introduce the space:

$$
M=H^{1}\left(\Omega ; \Gamma^{2}\right) .
$$

In the same way as in [1], we consider the following equivalent variational formulation of the problem (1.1)-(1.4):

Find $\mathbf{u}$ in $L^{2}(\Omega)^{2}$ and $p$ in $H^{1}(\Omega)$ such that $p-\varphi$ belongs to $M$ and that

$$
\begin{gathered}
\forall \mathbf{v} \in L^{2}(\Omega)^{2}, \quad a(\mathbf{u}, \mathbf{v})+b(\mathbf{v}, p)=\int_{\Omega} \mathbf{f}(\mathbf{x}) \cdot \mathbf{v}(\mathbf{x}) d \mathbf{x}, \\
\forall q \in M, \quad b(\mathbf{u}, q)=<U_{0}, q>_{\Gamma^{1}},
\end{gathered}
$$

where the bilinear forms $a$ and $b$ are defined by

$$
\begin{gathered}
\forall(\mathbf{v}, \mathbf{w}) \in\left(L^{2}(\Omega)^{2}\right)^{2}, \quad a(\mathbf{v}, \mathbf{w})=\int_{\Omega} \mathbf{v}(\mathbf{x}) \cdot \mathbf{w}(\mathbf{x}) d \mathbf{x}, \\
\forall \mathbf{v} \in L^{2}(\Omega)^{2}, \forall q \in H^{1}(\Omega), \quad b(\mathbf{v}, q)=\int_{\Omega} \mathbf{v}(\mathbf{x}) \cdot \nabla q(\mathbf{x}) d \mathbf{x} .
\end{gathered}
$$

Theorem 3.1 Let $\mathbf{f}$ be in $L^{2}(\Omega)^{2}, U_{0}$ in $H^{-1 / 2}\left(\Gamma^{1}\right)$ and $\varphi$ in $H^{1 / 2}\left(\Gamma^{2}\right)$, where $H^{-1 / 2}\left(\Gamma^{1}\right)$ and $H^{1 / 2}\left(\Gamma^{2}\right)$ are defined respectively in (2.3) and (2.2). Then problem (3.2), (3.3) has a unique solution satisfying

$$
\|\mathbf{u}\|_{L^{2}(\Omega)^{2}}+\|p\|_{H^{1}(\Omega)} \leq C\left(\|\mathbf{f}\|_{L^{2}(\Omega)}+\left\|U_{0}\right\|_{-1 / 2, \Gamma^{1}}+\|\varphi\|_{1 / 2, \Gamma^{2}}\right) .
$$

Proof. First, let us define $\tilde{\varphi}$ belonging to $H^{1 / 2}(\Gamma)$ such that $\tilde{\varphi}_{\mid \Gamma^{2}}=\varphi$ and $\|\tilde{\varphi}\|_{1 / 2, \Gamma} \leq$ $c\|\varphi\|_{1 / 2, \Gamma^{2}}$. To this end, we must extend $\varphi$ to a function belonging to $H^{1 / 2}(\Gamma)$. Let $\mu$ be a function defined in $[0,2]$ by

$$
\mu(t)=1-t, \text { for } 0 \leq t \leq 1 \text { and } \mu(t)=0, \text { for } 1 \leq t \leq 2 .
$$


We define $\tilde{\varphi}_{\mid \Gamma_{I I}}$ by

$$
\tilde{\varphi}\left(\mathbf{a}_{I}+t \boldsymbol{\tau}_{I I}\right)=\mu(t) \varphi\left(\mathbf{a}_{0}+(2-t) \boldsymbol{\tau}_{I}\right)+\mu(2-t) \varphi\left(\mathbf{a}_{I I}+(2-t) \boldsymbol{\tau}_{I I I}\right)
$$

and $\tilde{\varphi}_{\mid \Gamma_{I V}}$ by

$$
\tilde{\varphi}\left(\mathbf{a}_{O}-t \boldsymbol{\tau}_{I V}\right)=\mu(t) \varphi\left(\mathbf{a}_{0}+t \boldsymbol{\tau}_{I}\right)+\mu(2-t) \varphi\left(\mathbf{a}_{I I}+t \boldsymbol{\tau}_{I I I}\right) .
$$

Then we have $\|\tilde{\varphi}\|_{1 / 2, \Gamma} \leq C\|\varphi\|_{1 / 2, \Gamma^{2}}$. Next, let $\Phi$ in $H^{1}(\Omega)$ such that $\Phi_{\mid \Gamma}=\tilde{\varphi}$. Finally, we obtain a function $\Phi$ verifying

$$
\Phi_{\mid \Gamma^{2}}=\varphi \text { and }\|\Phi\|_{H^{1}(\Omega)} \leq C\|\varphi\|_{1 / 2, \Gamma^{2}} .
$$

Second, let us extend $U_{0}$ to a function belonging to $H^{-1 / 2}(\Gamma)$. We set $\tilde{U}_{0 \mid \Gamma^{1}}=U_{0}$ and $\tilde{U}_{0 \mid \Gamma^{2}}=-\frac{1}{2}<U_{0}, 1>_{\Gamma^{1}}$. Then, we have $\left\|\tilde{U}_{0}\right\|_{-1 / 2, \Gamma} \leq C\left\|U_{0}\right\|_{-1 / 2, \Gamma^{1}}$ and $<\tilde{U}_{0}, 1>_{\Gamma}=0$. Next, we define Neumann's Problem:

$$
\begin{cases}-\Delta \psi=0 & \text { in } \Omega \\ \frac{\partial \psi}{\partial n}=\tilde{U}_{0} & \text { on } \Gamma\end{cases}
$$

and we set $\mathbf{u}_{0}=\nabla \psi$. Applying Proposition 1.2 of [11, page 14], we derive that $\psi$ belongs to $H^{1}(\Omega)$, which implies that $\mathbf{u}_{0}$ belongs to $H(\operatorname{div} ; \Omega)$ with

$$
\left\|\mathbf{u}_{0}\right\|_{H(\operatorname{div} ; \Omega)}=|\psi|_{H^{1}(\Omega)} \leq C\left\|\tilde{U}_{0}\right\|_{-1 / 2, \Gamma}
$$

since $\operatorname{div} \mathbf{u}_{o}=\Delta \psi=0$. Finally, $\mathbf{u}_{0}$ verifies

$$
\mathbf{u}_{0} \cdot \mathbf{n}_{\mid \Gamma^{1}}=U_{0} \text {, div } \mathbf{u}_{0}=0 \text { in } \Omega \text { and }\left\|\mathbf{u}_{0}\right\|_{H(\operatorname{div} ; \Omega)} \leq C\left\|U_{0}\right\|_{-1 / 2, \Gamma^{1}} .
$$

Now, let us split $\mathrm{p}$ as: $p=\Phi+\tilde{p}$ with $\tilde{p}$ in $M$ and $\mathbf{u}$ as: $\mathbf{u}=\mathbf{u}_{0}+\tilde{\mathbf{u}}$. Then, we can write the problem $(3.2),(3.3)$ as

$$
\begin{gathered}
\forall \mathbf{v} \in L^{2}(\Omega)^{2}, \quad a(\tilde{\mathbf{u}}, \mathbf{v})+b(\mathbf{v}, \tilde{p})=\int_{\Omega}\left(\mathbf{f}(\mathbf{x})-\nabla \Phi(\mathbf{x})-\mathbf{u}_{0}(\mathbf{x})\right) \cdot \mathbf{v}(\mathbf{x}) d \mathbf{x} . \\
\forall q \in M, \quad b(\tilde{\mathbf{u}}, q)=0 .
\end{gathered}
$$

Since the right-hand side of (3.9) defines a continuous form on $L^{2}(\Omega)^{2}$ and since the properties of continuity and ellipticity are obvious we have only to check the following inf-sup condition on the form $b$ (see [11, pages 58,59]):

$$
\inf _{q \in M} \sup _{\mathbf{v} \in L^{2}(\Omega)^{2}} \frac{b(\mathbf{v}, q)}{\|v\|_{L^{2}(\Omega)^{2}}} \geq \beta \Longleftrightarrow \forall q \in M, \quad \sup _{\mathbf{v} \in L^{2}(\Omega)^{2}} \frac{b(\mathbf{v}, q)}{\|v\|_{L^{2}(\Omega)^{2}}} \geq \beta\|q\|_{H^{1}(\Omega)},
$$

with a positive constant $\beta$. This "inf-sup condition" was introduced independently by Babuska [3] and Brezzi [9]. We can verify this condition by taking $\mathbf{v}=\nabla q$. Indeed, we have

$$
\sup _{\mathbf{v} \in L^{2}(\Omega)^{2}} \frac{b(\mathbf{v}, q)}{\|v\|_{L^{2}(\Omega)^{2}}} \geq \frac{b(\nabla q, q)}{\|\nabla q\|_{L^{2}(\Omega)^{2}}}=|q|_{H^{1}(\Omega)}
$$

and, since $q_{\mid \Gamma^{2}}=0$, using a generalization of Poincaré inequality (see [11, Chap. I, page 40]) yields $\|q\|_{L^{2}(\Omega)} \leq \mathcal{P}|q|_{H^{1}(\Omega)}$, which implies

$$
\|q\|_{H^{1}(\Omega)} \leq \sqrt{\left.(\mathcal{P})^{2}+1\right)}|q|_{H^{1}(\Omega)} .
$$

Thus, the "inf-sup condition" is verified with the positive constant $\beta=\frac{1}{\sqrt{\left.(\mathcal{P})^{2}+1\right)}}$. Hence, applying Theorem 2.3 [7, pages 116,117], the theorem follows. 


\section{Second variational formulation}

We introduce the space:

$$
X=\left\{\mathbf{v} \in L^{2}(\Omega)^{2} ; \operatorname{div} \mathbf{v} \in L^{2}(\Omega) \text { and } \mathbf{v} \cdot \mathbf{n}_{\mid \Gamma^{1}}=0\right\} .
$$

In an analogous way as in [1], we consider the following equivalent variational formulation of the problem (1.1)-(1.4):

Find $\mathbf{u}$ in $\mathrm{X}$ and $p$ in $L^{2}(\Omega)$ such that $\mathbf{u}-\mathbf{u}_{0}$ belongs to $X$, where $\mathbf{u}_{0}$ is the function previously constructed that verifies (3.8), and that

$$
\begin{gathered}
\forall \mathbf{v} \in X, \quad a(\mathbf{u}, \mathbf{v})+b^{*}(\mathbf{v}, p)=\int_{\Omega} \mathbf{f}(\mathbf{x}) \cdot \mathbf{v}(\mathbf{x}) d \mathbf{x}-<\varphi, \mathbf{v} \cdot \mathbf{n}>_{\Gamma^{2}}, \\
\forall q \in L^{2}(\Omega), \quad b^{*}(\mathbf{u}, q)=0,
\end{gathered}
$$

where the bilinear form $b^{*}$ is defined by

$$
\forall \mathbf{v} \in H(\operatorname{div} ; \Omega), \forall q \in L^{2}(\Omega), \quad b^{*}(\mathbf{v}, q)=-\int_{\Omega} \operatorname{div} \mathbf{v}(\mathbf{x}) \cdot q(\mathbf{x}) d \mathbf{x} .
$$

In the same way as previously, we split $\mathbf{u}$ as: $\mathbf{u}=\mathbf{u}_{0}+\tilde{\mathbf{u}}$. Then, we can write the problem (3.13),(3.14) as

$$
\begin{gathered}
\forall \mathbf{v} \in X, \quad a(\tilde{\mathbf{u}}, \mathbf{v})+b^{*}(\mathbf{v}, p)=\int_{\Omega}\left(\mathbf{f}(\mathbf{x})-\mathbf{u}_{0}(\mathbf{x})\right) \cdot \mathbf{v}(\mathbf{x}) d \mathbf{x}-<\varphi, \mathbf{v} \cdot \mathbf{n}>_{\Gamma^{2}} . \\
\forall q \in L^{2}(\Omega), \quad b^{*}(\tilde{\mathbf{u}}, q)=0 .
\end{gathered}
$$

Since the right-hand side of (3.16) defines a continuous form on $X$ and since the properties of continuity and ellipticity are obvious, we have only to check the following inf-sup condition on the form $b^{*}$ :

$$
\forall q \in L^{2}(\Omega), \quad \sup _{\mathbf{v} \in X} \frac{b^{*}(\mathbf{v}, q)}{\|\mathbf{v}\|_{H(\operatorname{div} ; \Omega)}} \geq \beta^{*}\|q\|_{L^{2}(\Omega)},
$$

with a positive constant $\beta^{*}$. Let us note that $q_{0}=q-\frac{1}{|\Omega|} \int_{\Omega} q(\mathbf{x}) d \mathbf{x}$ belongs to $L_{0}^{2}(\Omega)$ and, owing to a classic result (see [11, Chap. I]), there exists $\mathbf{v}_{0}$ in $H_{0}^{1}(\Omega)^{2}$, such that

$$
\operatorname{div} \mathbf{v}_{0}=-q_{0} \quad \text { and } \quad\left\|\mathbf{v}_{0}\right\|_{H^{1}(\Omega)^{2}} \leq c\left\|q_{0}\right\|_{L^{2}(\Omega)} .
$$

Then, we set

$$
\tilde{\mathbf{v}}=\mathbf{v}_{0}+\mathbf{v}_{1} \quad \text { with } \quad \mathbf{v}_{1}(x, y)=\left(-\left(\frac{1}{|\Omega|} \int_{\Omega} q(\mathbf{x}) d \mathbf{x}\right) x, 0\right),-1 \leq x, y \leq 1 .
$$

We can verify that $\tilde{\mathbf{v}}$ belongs to $X$ with

$$
\operatorname{div} \tilde{\mathbf{v}}=-q \quad \text { and } \quad\|\tilde{\mathbf{v}}\|_{H(\operatorname{div} ; \Omega)} \leq C\|\tilde{\mathbf{v}}\|_{H^{1}(\Omega)^{2}} \leq C^{\prime}\|q\|_{L^{2}(\Omega)},
$$

since $\left|\int_{\Omega} q(\mathbf{x}) d \mathbf{x}\right| \leq \sqrt{|\Omega|}\|q\|_{L^{2}(\Omega}$. Then, we have

$$
\sup _{\mathbf{v} \in X} \frac{b^{*}(\mathbf{v}, q)}{\|\mathbf{v}\|_{H(\operatorname{div} ; \Omega)}} \geq \frac{b^{*}(\tilde{\mathbf{v}}, q)}{\|\tilde{\mathbf{v}}\|_{H(\operatorname{div} ; \Omega)}} \geq \frac{1}{C^{\prime}}\|q\|_{L^{2}(\Omega)} .
$$

Hence, we derive the inf-sup condition and we obtain the following result.

Theorem 3.2 Let $\mathbf{f}$ be in $L^{2}(\Omega)^{2}, U_{0}$ in $H^{-1 / 2}\left(\Gamma^{1}\right)$ and $\varphi$ in $H^{1 / 2}\left(\Gamma^{2}\right)$, where $H^{-1 / 2}\left(\Gamma^{1}\right)$ and $H^{1 / 2}\left(\Gamma^{2}\right)$ are defined respectively in (2.3) and (2.2). Then problem (3.13), (3.14) has a unique solution satisfying

$$
\|\mathbf{u}\|_{H(\operatorname{div}, \Omega)}+\|p\|_{L^{2}(\Omega)} \leq C\left(\|\mathbf{f}\|_{L^{2}(\Omega)}+\left\|U_{0}\right\|_{-1 / 2, \Gamma^{1}}+\|\varphi\|_{1 / 2, \Gamma^{2}}\right) .
$$




\section{Regularity results}

When the data $\mathbf{f}$ is in $H(\operatorname{div} ; \Omega)$, taking the divergence of the first equation of the problem (1.1)-(1.4) and owing to the other equations, we obtain a mixed problem of DirichletNeumann for the Laplace operator:

$$
\begin{cases}\Delta p=\operatorname{div} \mathbf{f} & \text { in } \Omega \\ p=\varphi & \text { on } \Gamma^{2} \\ \frac{\partial p}{\partial n}=\mathbf{f} \cdot \mathbf{n}-U_{0} & \text { on } \Gamma^{1}\end{cases}
$$

We suppose that $\mathbf{f}$ is in $H^{1}(\Omega)^{2}, \varphi$ is in $H^{3 / 2}\left(\Gamma^{2}\right)$ and $U_{0}$ is in $H^{1 / 2}\left(\Gamma^{1}\right)$. In addition, we assume matching conditions at the vertices of $\Gamma$ (see [7, Chap I]):

$$
\begin{gathered}
\int_{0}^{2}\left|\frac{d \varphi}{d \boldsymbol{\tau}_{J}}\left(\mathbf{a}_{J}-t \boldsymbol{\tau}_{J}\right)-\left(\mathbf{f} . \mathbf{n}-U_{0}\right)\left(\mathbf{a}_{J}+t \boldsymbol{\tau}_{J+1}\right)\right|^{2} \frac{d t}{t}<+\infty, J=I, I I I \\
\int_{0}^{2}\left|\left(\mathbf{f} \cdot \mathbf{n}-U_{0}\right)\left(\mathbf{a}_{J}-t \boldsymbol{\tau}_{J}\right)+\frac{d \varphi}{d \boldsymbol{\tau}_{J+1}}\left(\mathbf{a}_{J}+t \boldsymbol{\tau}_{J+1}\right)\right|^{2} \frac{d t}{t}<+\infty, J=I I, I V .
\end{gathered}
$$

Theorem 3.3 For any data $\mathbf{f}$ in $H^{1}(\Omega)^{2}, \varphi$ in $H^{3 / 2}\left(\Gamma^{2}\right)$ and $U_{0}$ in $H^{1 / 2}\left(\Gamma^{1}\right)$, where $H^{3 / 2}\left(\Gamma^{2}\right)$ and $H^{1 / 2}\left(\Gamma^{1}\right)$ are defined in (2.2), verifying the matching conditions (3.21), the solution $(\mathbf{u}, p)$ of the problem (1.1)-(1.4) belongs to $H^{1}(\Omega)^{2} \times H^{2}(\Omega)$.

Proof. Owing to matching conditions $(3.21)$, there exists $p_{0}$ in $H^{2}(\Omega)$ such that $p_{0 \mid \Gamma^{2}}=\varphi$ and $\left(\frac{\partial p_{0}}{\partial n}\right)_{\mid \Gamma^{1}}=\mathbf{f} . \mathbf{n}-U_{0}$. Let us set $\tilde{p}=p-p_{0}$. The problem (3.20) is equivalent to the following problem: find $\tilde{p}$ in $H^{1}\left(\Omega ; \Gamma^{2}\right)$ such that

$$
\forall q \in H^{1}\left(\Omega ; \Gamma^{2}\right), a(\nabla \tilde{p}, \nabla q)=\int_{\Omega}\left(\operatorname{div} \mathbf{f}+\Delta p_{0}\right)(\mathbf{x}) q(\mathbf{x}) d \mathbf{x} .
$$

Since the boundary between $\Gamma^{1}$ and $\Gamma^{2}$ is the set of vertices of $\Gamma$, the regularity of the data implies that this homogeneous mixed problem of Dirichlet-Neumann for the Laplace operator has a solution $\tilde{p}$ in $H^{2}(\Omega)$ (see [12]). Hence, we derive the regularity of $p$ and $\mathbf{u} . \diamond$

Remark 3.4 If (f . $\left.\mathbf{n}-U_{0}\right)$ is Lipschitz-continuous on $\overline{\Gamma_{J}}$ or belongs to $H^{1}\left(\Gamma_{J}\right), J=I I, I V$ and if $\varphi$ belongs to $C^{1,1}\left(\overline{\Gamma_{J}}\right)$ or to $H^{2}\left(\Gamma_{J}\right), J=I, I I I$, the matching conditions (3.21) are equivalent to simpler conditions:

$\frac{d \varphi}{d \boldsymbol{\tau}_{J}}\left(\mathbf{a}_{J}\right)=\left(\mathbf{f} \cdot \mathbf{n}-U_{0}\right)\left(\mathbf{a}_{J}\right), J=I, I I I$ and $-\frac{d \varphi}{d \boldsymbol{\tau}_{J+1}}\left(\mathbf{a}_{J}\right)=\left(\mathbf{f} \cdot \mathbf{n}-U_{0}\right)\left(\mathbf{a}_{J}\right), J=I I, I V$.

\section{SPECTRAL DISCRETIZATION}

\section{First spectral discretization}

We define the discrete scalar product by

$$
(u, v)_{N}=\sum_{i=0}^{N} \sum_{j=0}^{N} u\left(\xi_{i}, \xi_{j}\right) v\left(\xi_{i}, \xi_{j}\right) \rho_{i} \rho_{j}
$$

and we denote by $\mathcal{I}_{N}$ the Lagrange interpolation operator at the points $\left(\xi_{i}, \xi_{j}\right), 0 \leq i, j \leq N$ in $\mathbb{P}_{N}(\Omega)$. We set

$$
X_{N}=\mathbb{P}_{N}(\Omega)^{2} \quad \text { or } \quad X_{N}=\left(\mathbb{P}_{N-1}(\Lambda) \otimes \mathbb{P}_{N}(\Lambda)\right) \times\left(\mathbb{P}_{N}(\Lambda) \otimes \mathbb{P}_{N-1}(\Lambda)\right) .
$$


We assume that the data $\mathbf{f}$ belongs to $C^{0}(\bar{\Omega})^{2}$ and, for the sake of simplicity, that $\mathbf{u}$ and $p$ satisfy homogeneous boundary conditions, that is to say, we set $\varphi=0, U_{0}=0$ in (1.1)-(1.4). From the variational formulation (3.2)-(3.3), we derive the following discrete problem:

Find $\mathbf{u}_{N}$ in $X_{N}$ and $p_{N}$ in $\mathbb{P}_{N}(\Omega) \cap M$, where $M$ is defined by (3.1), such that

$$
\begin{array}{r}
\forall \mathbf{v}_{N} \in X_{N}, \quad\left(\mathbf{u}_{N}, \mathbf{v}_{N}\right)_{N}+b_{N}\left(\mathbf{v}_{N}, p_{N}\right)=\left(\mathbf{f}, \mathbf{v}_{N}\right)_{N}, \\
\forall q_{N} \in \mathbb{P}_{N}(\Omega) \cap M, \quad b_{N}\left(\mathbf{u}_{N}, q_{N}\right)=0,
\end{array}
$$

where the form $b_{N}$ is defined by

$$
\forall \mathbf{v}_{N} \in \mathbb{P}_{N}(\Omega)^{2}, \forall q_{N} \in \mathbb{P}_{N}(\Omega), b_{N}\left(\mathbf{v}_{N}, q_{N}\right)=\left(\mathbf{v}_{N}, \nabla q_{N}\right)_{N} .
$$

We have a classical saddle point problem. We verify the inf-sup condition

$$
\forall q_{N} \in \mathbb{P}_{N}(\Omega) \cap M, \quad \sup _{\mathbf{v}_{N} \in X_{N}} \frac{b_{N}\left(\mathbf{v}_{N}, q_{N}\right)}{\left\|v_{N}\right\|_{L^{2}(\Omega)^{2}}} \geq \gamma\left\|q_{N}\right\|_{H^{1}(\Omega)},
$$

where $\gamma$ is a positive constant independent from $N$, by taking $\mathbf{v}_{N}=\nabla q_{N}$. Hence, we derive the following theorem.

Theorem 4.1 Let $\mathbf{f}$ be in $C^{0}(\bar{\Omega})^{2}$. Then problem (4.3), (4.4) has a unique solution $\left(\mathbf{u}_{N}, p_{N}\right)$ satisfying

$$
\left\|\mathbf{u}_{N}\right\|_{L^{2}(\Omega)^{2}}+\left\|p_{N}\right\|_{H^{1}(\Omega)} \leq C\left\|\mathcal{I}_{N} \mathbf{f}\right\|_{L^{2}(\Omega)^{2}} .
$$

Next, we establish a theorem which implies the convergence of our discretization method.

Theorem 4.2 Assume that the solution ( $\mathbf{u}, p)$ of problem (4.3), (4.4) belongs to $H^{s}(\Omega)^{2} \times$ $H^{s+1}(\Omega), s \geq 0$, and the data $\mathbf{f}$ belongs to $H^{\sigma}(\Omega)^{2}, \sigma>1$, where $H^{s}(\Omega)$, for non-integral values of $s$, is defined in (2.1). Then, the following estimate holds

$$
\left\|\mathbf{u}-\mathbf{u}_{N}\right\|_{L^{2}(\Omega)^{2}}+\left\|p-p_{N}\right\|_{H^{1}(\Omega)} \leq c\left(N^{-s}\left(\|\mathbf{u}\|_{H^{s}(\Omega)^{2}}+\|p\|_{H^{s+1}(\Omega)}\right)+N^{-\sigma}\|\mathbf{f}\|_{H^{\sigma}(\Omega)^{2}}\right) .
$$

Proof. From the abstract error estimate for the approximation of saddle-point problems (see [7, Chap. IV]), we derive the following estimate:

$$
\begin{array}{r}
\left\|\mathbf{u}-\mathbf{u}_{N}\right\|_{L^{2}(\Omega)^{2}}+\left\|p-p_{N}\right\|_{H^{1}(\Omega)} \leq c\left(\inf _{\mathbf{w}_{N} \in V_{N}}\left\|\mathbf{u}-\mathbf{w}_{N}\right\|_{L^{2}(\Omega)^{2}}\right. \\
+\inf _{\mathbf{v}_{N} \in X_{N}}\left(\left\|\mathbf{u}-\mathbf{v}_{N}\right\|_{L^{2}(\Omega)^{2}}+\sup _{\mathbf{z}_{N} \in X_{N}} \frac{\int_{\Omega} \mathbf{v}_{N}(\mathbf{x}) \cdot \mathbf{z}_{N}(\mathbf{x}) d \mathbf{x}-\left(\mathbf{v}_{N}, \mathbf{z}_{N}\right)_{N}}{\left\|\mathbf{z}_{N}\right\|_{L^{2}(\Omega)^{2}}}\right) \\
+\inf _{q_{N} \in \mathbb{P}_{N}(\Omega) \cap M}\left(\left\|p-q_{N}\right\|_{H^{1}(\Omega)}+\sup _{\mathbf{z}_{N} \in X_{N}} \frac{b\left(\mathbf{z}_{N}, q_{N}\right)-b_{N}\left(\mathbf{z}_{N}, q_{N}\right)}{\left\|\mathbf{z}_{N}\right\|_{L^{2}(\Omega)^{2}}}\right) \\
\left.+\sup _{\mathbf{z}_{N} \in X_{N}} \frac{\int_{\Omega} \mathbf{f}(\mathbf{x}) \cdot \mathbf{z}_{N}(\mathbf{x}) d \mathbf{x}-\left(\mathbf{f}, \mathbf{z}_{N}\right)_{N}}{\left\|\mathbf{z}_{N}\right\|_{L^{2}(\Omega)^{2}}}\right),
\end{array}
$$

where $V_{N}$ is defined by

$$
V_{N}=\left\{\mathbf{w}_{N} \in X_{N} ; \forall q_{N} \in \mathbb{P}_{N}(\Omega) \cap M, b_{N}\left(\mathbf{w}_{N}, q_{N}\right)=0\right\} .
$$

Moreover, we recall (see [11, Chap. II, (1.16)]) that

$$
\inf _{\mathbf{w}_{N} \in V_{N}}\left\|\mathbf{u}-\mathbf{w}_{N}\right\|_{L^{2}(\Omega)^{2}} \leq \frac{c}{\gamma} \inf _{\mathbf{v}_{N} \in X_{N}}\left(\left\|\mathbf{u}-\mathbf{v}_{N}\right\|_{L^{2}(\Omega)^{2}}\right)
$$

Hence, we derive that, for all $\mathbf{v}_{N-1}$ and $\mathbf{f}_{N-1}$ in $\mathbb{P}_{N-1}(\Omega)^{2}$ and all $q_{N-1} \in \mathbb{P}_{N-1}(\Omega) \cap M$,

$$
\begin{gathered}
\left\|\mathbf{u}-\mathbf{u}_{N}\right\|_{L^{2}(\Omega)^{2}}+\left\|p-p_{N}\right\|_{H^{1}(\Omega)} \\
\leq c\left(\left\|\mathbf{u}-\mathbf{v}_{N-1}\right\|_{L^{2}(\Omega)^{2}}+\left\|p-q_{N-1}\right\|_{H^{1}(\Omega)}+\left\|\mathbf{f}-\mathbf{f}_{N-1}\right\|_{L^{2}(\Omega)^{2}}+\left\|\mathbf{f}-\mathcal{I}_{N} \mathbf{f}\right\|_{L^{2}(\Omega)^{2}}\right) .
\end{gathered}
$$


Then, we choose $\mathbf{v}_{N-1}=\Pi_{N-1} \mathbf{u}$ (resp. $\mathbf{f}_{N-1}=\Pi_{N-1} \mathbf{f}$ ), that is to say the orthogonal projection of $\mathbf{u}$ (resp. f) on $\mathbb{P}_{N-1}(\Omega)^{2}$ in $L^{2}(\Omega)^{2}$ and $q_{N-1}=\Pi_{N-1}^{1, \Gamma_{2}} p$, where $\Pi_{N-1}^{1, \Gamma_{2}} p$ is the orthogonal projection of $p$ on $\mathbb{P}_{N-1}(\Omega) \cap M$ in $H^{1}(\Omega)$. It remains to prove the estimate, for any $m \geq 1$,

$$
\forall p \in H^{m}(\Omega) \cap M,\left\|p-\Pi_{N-1}^{1, \Gamma_{2}} p\right\|_{H^{1}(\Omega)} \leq C N^{1-m}\|p\|_{H^{m}(\Omega)} .
$$

On the one hand, this result is obvious for $m=1$. On the other hand, for $m \geq 2$, we have (see [7, Chap. III]),

$$
\begin{array}{r}
\left\|p-\Pi_{N-1}^{1, \Gamma_{2}} p\right\|_{H^{1}(\Omega)} \leq \inf _{r_{N-1} \in \mathbb{P}_{N-1}(\Omega) \cap M}\left\|p-r_{N-1}\right\|_{H^{1}(\Omega)} \\
\leq\left\|p-\mathcal{I}_{N-1} p\right\|_{H^{1}(\Omega)} \leq C N^{1-m}\|p\|_{H^{m}(\Omega)} .
\end{array}
$$

Then, an interpolation argument (see [11, TH 1.4, page 6]) gives (4.11). Finally, the result follows from (4.10), (4.11) and the classic estimate for the orthogonal projection on $\mathbb{P}_{N-1}(\Omega)$ in $L^{2}(\Omega)$.

Remark 4.3 With the choice $X_{N}=\mathbb{P}_{N}(\Omega)^{2}$, problem (4.3, (4.4) can be interpreted as a collocation scheme. Indeed, by integrating by parts in the discrete bilinear form $b_{N}$ with respect to one of the two variables for each of the two terms of $b_{N}$ (this process being allowed by the precision of the quadrature rule), and choosing as test functions the Lagrange polynomials associated with the grid points of $\Xi_{N}$, it is easily seen that (4.3), (4.4) is equivalent to the set of equations for $\mathbf{u}_{N}$ in $l P_{N}(\Omega)^{2}$ and $p_{N}$ in $P_{N}(\Omega) \cap M$ :

$$
\begin{array}{cr}
\mathbf{u}_{N}(\mathbf{x})+\nabla p_{N}(\mathbf{x})=\mathbf{f}(\mathbf{x}), & \forall \mathbf{x} \in \Xi_{N}, \\
\operatorname{div} \mathbf{u}_{N}(\mathbf{x})=0, & \forall \mathbf{x} \in \Xi_{N} \cap \Omega, \\
\frac{2}{N(N+1)} \operatorname{div} \mathbf{u}_{N}(\mathbf{x})=\left(\mathbf{u}_{N} \cdot \mathbf{n}\right)(\mathbf{x}), & \forall \mathbf{x} \in \Xi_{N} \cap \Gamma^{1} .
\end{array}
$$

\section{Second spectral discretization}

In order to improve the approximation of the condition $\operatorname{div} \mathbf{u}=0$, we can try to decrease the dimension of the space $X_{N}$. So, we choose

$$
X_{N}=\mathbb{P}_{N-1}(\Omega)^{2} \text {. }
$$

We note that, in this case the forms $b(.,$.$) and b_{N}(.,$.$) are equal on X_{N} \times \mathbb{P}_{N}(\Omega)$. It does not appear spurious modes for the pressure, as we can see in the following lemma.

Lemma 4.4 Let $\mathcal{Z}_{N}$ be the space

$$
\mathcal{Z}_{N}=\left\{q_{N} \in \mathbb{P}_{N}(\Omega) \cap M ; \forall \mathbf{v}_{N} \in \mathbb{P}_{N-1}(\Omega)^{2}, b\left(\mathbf{v}_{N}, q_{N}\right)=0\right\} .
$$

Then $\mathcal{Z}_{N}=\{0\}$.

Proof. Let $q_{N}$ be in $\mathcal{Z}_{N}$. Since $\frac{\partial q_{N}}{\partial x}$ is a polynomial of degree $\leq N-1$ with respect to $x$, which is orthogonal to $\mathbb{P}_{N-1}(\Omega)$, we can write:

$$
q_{N}(x, y)=\alpha_{N}(y)+\beta_{N}(x) L_{N}(y),
$$

with $\alpha_{N}$ and $\beta_{N}$ in $\mathbb{P}_{N}(\Omega)$. In the same way with $y$ in place of $x$, we have:

$$
q_{N}(x, y)=\gamma_{N}(x)+\delta_{N}(y) L_{N}(x),
$$

with $\gamma_{N}$ and $\delta_{N}$ in $\mathbb{P}_{N}(\Omega)$. Hence, we derive

$$
q_{N}(x, y)=\lambda+\mu L_{N}(x) L_{N}(y),
$$


where $\lambda$ and $\mu$ are real numbers. But, since $L_{N}(1)=0$, the condition:

$$
\forall y \in[-1,1], q_{N}(1, y)=0
$$

implies $\lambda=\mu=0$.

We have to study the following discrete problem:

Find $\mathbf{u}_{N}$ in $\mathbb{P}_{N-1}(\Omega)^{2}$ and $p_{N}$ in $\mathbb{P}_{N}(\Omega) \cap M$ such that

$$
\begin{array}{r}
\forall \mathbf{v}_{N} \in \mathbb{P}_{N-1}(\Omega)^{2}, \quad\left(\mathbf{u}_{N}, \mathbf{v}_{N}\right)_{N}+b\left(\mathbf{v}_{N}, p_{N}\right)=\left(\mathbf{f}, \mathbf{v}_{N}\right)_{N}, \\
\forall q_{N} \in \mathbb{P}_{N}(\Omega) \cap M, \quad b\left(\mathbf{u}_{N}, q_{N}\right)=0 .
\end{array}
$$

For the inf-sup condition, the choice $\mathbf{v}_{N}=\nabla q_{N}$ is no longer available, since $\mathbf{v}_{N}$ must be in $\mathbb{P}_{N-1}(\Omega)^{2}$. In fact, in the next lemma, we find a inf-sup constant depending on $N$.

Lemma 4.5 There exists a constant $c>0$ independent on $N$ such that

$$
\forall q_{N} \in \mathbb{P}_{N}(\Omega) \cap H^{1}\left(\Omega ; \Gamma^{2}\right), \quad \sup _{\mathbf{v}_{N} \in \mathbb{P}_{N-1}(\Omega)^{2}} \frac{b\left(\mathbf{v}_{N}, q_{N}\right)}{\left\|\mathbf{v}_{N}\right\|_{L^{2}(\Omega)^{2}}} \geq c N^{-1}\left\|q_{N}\right\|_{H^{1}(\Omega)} .
$$

On the one hand, we note that

$$
q_{N}^{*}(x, y)=\sum_{n=0}^{N} a_{n}(x) L_{n}(y),
$$

where $a_{N}$ is a polynomial of degree $\leq N-1$. Then, we have

$$
\frac{\partial q_{N}^{*}}{\partial x}(x, y)=\Pi_{N-1}\left(\frac{\partial q_{N}^{*}}{\partial x}\right)(x, y)+a_{N}^{\prime}(x) L_{N}(y),
$$

which implies, using (2.7) and the inverse inequality (2.10),

$$
\left\|\frac{\partial q_{N}^{*}}{\partial x}\right\|_{L^{2}(\Omega)} \leq\left\|\Pi_{N-1}\left(\frac{\partial q_{N}^{*}}{\partial x}\right)\right\|_{L^{2}(\Omega)}+c N^{\frac{3}{2}}\left\|a_{N}\right\|_{0, \Lambda} .
$$

On the other hand, in view of (2.8), we can write

$$
\Pi_{N-1}\left(\frac{\partial q_{N}^{*}}{\partial y}\right)\left((x, y)=\sum_{n=0}^{N} \Pi_{N-1}\left(a_{n} L_{n}^{\prime}\right)(x, y)=(2 N-1) a_{N}(x) L_{N-1}(y)+r_{N}(x, y),\right.
$$

where $r_{N}(x, y)$ is a polynomial of $\mathbb{P}_{N}(\Omega)$ of degree $<N-1$ with respect to $y$. The orthogonality properties imply

$$
\left\|\Pi_{N-1}\left(\frac{\partial q_{N}^{*}}{\partial y}\right)\right\|_{L^{2}(\Omega)} \geq 2 \sqrt{N-\frac{1}{2}}\left\|a_{N}\right\|_{0, \Lambda} .
$$

By combining this inequality with (4.18), we obtain

$$
\left\|\frac{\partial q_{N}^{*}}{\partial x}\right\|_{L^{2}(\Omega)} \leq\left\|\Pi_{N-1}\left(\frac{\partial q_{N}^{*}}{\partial x}\right)\right\|_{L^{2}(\Omega)}+c N\left\|\Pi_{N-1}\left(\frac{\partial q_{N}^{*}}{\partial y}\right)\right\|_{L^{2}(\Omega)} .
$$

In the same way, we have the analogous inequality for $\frac{\partial q_{N}^{*}}{\partial y}$. Thus, we obtain

$$
\left\|\nabla q_{N}^{*}\right\|_{L^{2}(\Omega)^{2}} \leq c N\left\|\Pi_{N-1}\left(\nabla q_{N}^{*}\right)\right\|_{L^{2}(\Omega)^{2}} .
$$

Next, the equality (4.16) yields

$$
\left\|\nabla q_{N}\right\|_{L^{2}(\Omega)} \leq\left\|\nabla q_{N}^{*}\right\|_{L^{2}(\Omega)}+\left|\alpha_{N}\right|\left\|\nabla\left(L_{N}(x) L_{N}(y)\right)\right\|_{L^{2}(\Omega)},
$$

which implies, owing to (2.7) and (2.9), 


$$
\left\|\nabla q_{N}\right\|_{L^{2}(\Omega)} \leq\left\|\nabla q_{N}^{*}\right\|_{L^{2}(\Omega)}+2 \sqrt{\frac{N(N+1)}{2 N+1}}\left|\alpha_{N}\right| .
$$

It remains to estimate $\left|\alpha_{N}\right|$. First, we have, owing to (4.16),

$$
q_{N}(x, y)=\sum_{n=0}^{N} a_{n}(x) L_{n}(y)+\alpha_{N} L_{N}(x) L_{N}(y) .
$$

But, $\forall y \in[-1,1], q_{N}(1, y)=0$, which implies

$$
a_{n}(1)=0, n=1, \ldots, N-1 \quad \text { and } \quad a_{N}(1)+\alpha_{N}=0 .
$$

If we set $a_{N}(x)=\sum_{k=1}^{N-1} \alpha_{k} L_{k}(x)$, we derive

$$
\alpha_{N}=-\sum_{k=0}^{N-1} \alpha_{k}
$$

and, therefore, thanks to a discrete Cauchy-Schwarz inequality

$$
\left|\alpha_{N}\right| \leq\left(\sum_{k=0}^{N-1} \frac{\alpha_{k}^{2}}{k+\frac{1}{2}}\right)^{\frac{1}{2}}\left(\sum_{k=0}^{N-1}\left(k+\frac{1}{2}\right)\right)^{\frac{1}{2}} \leq \frac{\sqrt{2}}{2} N\left\|a_{N}\right\|_{0, \Lambda} .
$$

Then, in view of (4.19), we obtain

$$
\left|\alpha_{N}\right| \leq \frac{\sqrt{2} N}{4 \sqrt{N-\frac{1}{2}}}\left\|\Pi_{N-1}\left(\nabla q_{N}^{*}\right)\right\|_{L^{2}(\Omega)} .
$$

Finally, (4.20), (4.21) and (4.22) yield

$$
\left\|\nabla q_{N}\right\|_{L^{2}(\Omega)^{2}} \leq c N\left\|\Pi_{N-1}\left(\nabla q_{N}^{*}\right)\right\|_{L^{2}(\Omega)}
$$

which, in view of (4.17), ends the proof.

The bilinear form $(., .)_{N}$ and $b(.,$.$) satisfy Brezzi's conditions with respect to \mathbb{P}_{N-1}(\Omega)^{2}$ and $\mathbb{P}_{N}(\Omega) \cap M$ (the bilinear form $(.,)_{N}$ is continuous on $\mathbb{P}_{N-1}(\Omega)^{2}$ and elliptic on $\mathbb{P}_{N-1}(\Omega)$, the bilinear form $b(.,$.$) is continuous on \mathbb{P}_{N-1}(\Omega) \times\left(\mathbb{P}_{N}(\Omega) \cap M\right)$ and verifies the "inf-sup condition"), see [7, Theorem 2.3, pages 116,117], whence the theorem.

Theorem 4.6 Let $\mathbf{f}$ be in $C^{0}(\bar{\Omega})^{2}$. Then problem (4.13), (4.14) has a unique solution $\left(\mathbf{u}_{N}, p_{N}\right)$ satisfying

$$
\left\|\mathbf{u}_{N}\right\|_{L^{2}(\Omega)^{2}}+N^{-1}\left\|p_{N}\right\|_{H^{1}(\Omega)} \leq C\left\|\mathcal{I}_{N} \mathbf{f}\right\|_{L^{2}(\Omega)^{2}} .
$$

We establish the convergence of this second discretization in the following theorem.

Theorem 4.7 Assume that the solution $(\mathbf{u}, p)$ of problem (4.13), (4.14) belongs to $H^{s}(\Omega)^{2}$ $\times H^{s+1}(\Omega), s \geq 0$, and the data $\mathbf{f}$ belongs to $H^{\sigma}(\Omega)^{2}, \sigma>1$. Then, the following estimate holds

$$
\left\|\mathbf{u}-\mathbf{u}_{N}\right\|_{L^{2}(\Omega)^{2}}+N^{-1}\left\|p-p_{N}\right\|_{H^{1}(\Omega)} \leq c\left(N^{-s}\left(\|\mathbf{u}\|_{H^{s}(\Omega)^{2}}+\|p\|_{H^{s+1}(\Omega)}\right)+N^{-\sigma}\|\mathbf{f}\|_{H^{\sigma}(\Omega)^{2}}\right) .
$$

Proof. The abstract error estimate, analogous to (4.39) but with much simplification because of the exactness of the quadrature formulas, yields

$$
\left\|\mathbf{u}-\mathbf{u}_{N}\right\|_{L^{2}(\Omega)^{2}}+N^{-1}\left\|p-p_{N}\right\|_{H^{1}(\Omega)} \leq c\left(\inf _{\mathbf{w}_{N} \in V_{N}}\left\|\mathbf{u}-\mathbf{w}_{N}\right\|_{L^{2}(\Omega)^{2}}\right.
$$




$$
\left.+\inf _{\mathbf{v}_{N} \in \mathbb{P}_{N-1}(\Omega)^{2}}\left\|\mathbf{u}-\mathbf{v}_{N}\right\|_{L^{2}(\Omega)^{2}}+\inf _{q_{N} \in \mathbb{P}_{N}(\Omega) \cap M}\left\|p-q_{N}\right\|_{H^{1}(\Omega)}+\left\|\mathbf{f}-\mathcal{I}_{N} \mathbf{f}\right\|_{L^{2}(\Omega)^{2}}\right),
$$

where $V_{N}$ is now the space

$$
V_{N}=\left\{\mathbf{w}_{N} \in \mathbb{P}_{N-1}(\Omega)^{2} ; \forall q_{N} \in \mathbb{P}_{N}(\Omega) \cap M, b\left(\mathbf{w}_{N}, q_{N}\right)=0\right\} .
$$

It remains to estimate the term $\inf _{\mathbf{w}_{N} \in V_{N}}\left\|\mathbf{u}-\mathbf{w}_{N}\right\|_{L^{2}(\Omega)^{2}}$. Since $\mathbf{u}$ is such that $\operatorname{div} \mathbf{u}=0$ and $\mathbf{u} \cdot \mathbf{n}_{\mid \Gamma^{1}}=0$, there exist a unique $\psi$ in $H^{1}(\Omega)$ (see [11, Chap. I and 4]) such that

$$
\mathbf{u}=\operatorname{curl} \psi \quad \text { and } \quad \psi=0 \text { on } \Gamma^{1} .
$$

Moreover, if $\mathbf{u}$ belongs to $H^{s}(\Omega)^{2}$, we have $\|\psi\|_{H^{s+1}(\Omega)} \leq c\|\mathbf{u}\|_{H^{s}(\Omega)^{2}}$. Let us define the operator $\tilde{\pi}_{N}^{1}$ (see [7, Chap. II]) on $H^{1}(\Lambda)$ by

$$
\forall \varphi \in H^{1}(\Lambda),\left(\tilde{\pi}_{N}^{1} \varphi\right)(\zeta)=\left(\pi_{N}^{1,0} \tilde{\varphi}\right)(\zeta)+\varphi(-1) \frac{1-\zeta}{2}+\varphi(1) \frac{1+\zeta}{2},
$$

where the function $\tilde{\varphi}$ stands for

$$
\tilde{\varphi}(\zeta)=\varphi(\zeta)-\varphi(-1) \frac{1-\zeta}{2}-\varphi(1) \frac{1+\zeta}{2} .
$$

Note that the definition of $\tilde{\pi}_{N}^{1}$ is available, because $\tilde{\varphi}$ belongs to $H_{0}^{1}(\Lambda)$, and that $\pi_{N}^{1,0} \tilde{\varphi}$ and $\varphi$ coincide in -1 and 1 . In [8, Section 7], the following estimate is proven, for all $r \geq 1$ and all function $\varphi$ in $H^{r}(\Lambda)$ :

$$
\left|\varphi-\tilde{\pi}_{N}^{1} \varphi\right|_{1, \Lambda}+N\left\|\varphi-\tilde{\pi}_{N}^{1} \varphi\right\|_{0, \Lambda} \leq c N^{1-r}\|\varphi\|_{r, \Lambda} .
$$

Assuming $s \geq 1$, we set

$$
R_{N-1}(\mathbf{u})=\operatorname{curl}\left(\tilde{\pi}_{N-1}^{1(x)} \circ \tilde{\pi}_{N-1}^{1(y)} \psi\right) .
$$

Since $\left(\tilde{\pi}_{N-1}^{1(x)} \circ \tilde{\pi}_{N-1}^{1(y)} \psi\right)_{\mid \Gamma^{1}}=\psi_{\mid \Gamma^{1}}=0$, we can verify that $R_{N-1}(\mathbf{u})$ belongs to $V_{N}$ and, in view of (4.26), that

$$
\left|\varphi-\tilde{\pi}_{N-1}^{1(x)} \circ \tilde{\pi}_{N-1}^{1(y)} \psi\right|_{H^{1}(\Omega)} \leq c N^{-s}\|\psi\|_{H^{s+1}(\Omega)} .
$$

Finally, we derive, for $s \geq 1$,

$$
\inf _{\mathbf{w}_{N} \in V_{N}}\left\|\mathbf{u}-\mathbf{w}_{N}\right\|_{L^{2}(\Omega)^{2}} \leq\left\|\mathbf{u}-R_{N-1}(\mathbf{u})\right\|_{L^{2}(\Omega)^{2}} \leq c N^{-s}\|\mathbf{u}\|_{H^{s}(\Omega)^{2}} .
$$

Since we have $\inf _{\mathbf{w}_{N} \in V_{N}}\left\|\mathbf{u}-\mathbf{w}_{N}\right\|_{L^{2}(\Omega)^{2}} \leq c\|\mathbf{u}\|_{L^{2}(\Omega)}$, an interpolation argument gives the result of approximation in $V_{N}$ for any $s \geq 0$ and the estimate of the theorem follows.

\section{Third spectral discretization}

The third discretization comes from the variational formulation (3.13), (3.14). We define the space $X_{N}$ by

$$
X_{N}=\mathbb{P}_{N}(\Omega)^{2} \cap X=\left\{\mathbf{v}_{N} \in \mathbb{P}_{N}(\Omega)^{2} ; \mathbf{v}_{N} \cdot \mathbf{n}_{\mid \Gamma^{1}}=0\right\} .
$$

Let $M_{N}$ be a subspace of $\mathbb{P}_{N}(\Omega)$ that we shall set later. Then, we consider the following discrete problem:

Find $\mathbf{u}_{N}$ in $X_{N}$ and $p_{N}$ in $M_{N}$ such that

$$
\begin{array}{r}
\forall \mathbf{v}_{N} \in X_{N}, \quad\left(\mathbf{u}_{N}, \mathbf{v}_{N}\right)_{N}+b_{N}^{*}\left(\mathbf{v}_{N}, p_{N}\right)=\left(\mathbf{f}, \mathbf{v}_{N}\right)_{N}, \\
\forall q_{N} \in M_{N}, \quad b_{N}^{*}\left(\mathbf{u}_{N}, q_{N}\right)=0,
\end{array}
$$


where the form $b_{N}^{*}$ is define by

$$
\forall \mathbf{v}_{N} \in \mathbb{P}_{N}(\Omega)^{2}, \forall q_{N} \in \mathbb{P}_{N}(\Omega), b_{N}^{*}\left(\mathbf{v}_{N}, q_{N}\right)=-\left(\operatorname{div} \mathbf{v}_{N}, q_{N}\right)_{N}
$$

In order to choose $M_{N}$, we begin to identify the spurious modes for the pressure. These spurious modes for the pressure are derived by elimination from those of classic Stokes problem (see [7, Chap. IV]). In particular, we can verify: $\forall \mathbf{v}_{N} \in X_{N}, b_{N}^{*}\left(\mathbf{v}_{N}, q_{N}\right)=0$, for $q_{N}(x, y)=L_{N}(x)$ or $L_{N}(x) L_{N}(y)$. We obtain the following lemma.

Lemma 4.8 Let $\mathcal{Z}_{N}^{*}$ be the space

$$
\mathcal{Z}_{N}^{*}=\left\{q_{N} \in P_{N}(\Omega) ; \forall \mathbf{v}_{N} \in X_{N}, b_{N}^{*}\left(\mathbf{v}_{N}, q_{N}\right)=0\right\} .
$$

Then $\mathcal{Z}_{N}^{*}$ is spanned by $\left(L_{N}(x), L_{N}(x) L_{N}(y)\right)$.

Finally, let $M_{N}$ stand for the orthogonal complement of $\mathcal{Z}_{N}^{*}$ for the scalar product in $L^{2}(\Omega)$ or for the scalar product $(., .)_{N}$, owing to $(2.11)$. The inf-sup condition is given in the next lemma.

Lemma 4.9 There exists a constant $c>0$ independent from $N$ such that

$$
\forall q_{N} \in M_{N}, \quad \sup _{\mathbf{v}_{N} \in X_{N}} \frac{b_{N}^{*}\left(\mathbf{v}_{N}, q_{N}\right)}{\left\|v_{N}\right\|_{H(\operatorname{div} ; \Omega)}} \geq c\left\|q_{N}\right\|_{L^{2}(\Omega)} .
$$

Proof. Any function $q_{N}$ in $M_{N}$ has the expansion

$$
\begin{gathered}
q_{N}(x, y)=\sum_{m=0}^{N-1} \sum_{n=0}^{N-1} q_{m, n} L_{m}(x) L_{n}(y) \\
+\sum_{m=0}^{N-1} q_{m, N} L_{m}(x)\left(L_{N}(y)-L_{N-2}(y)\right)+\sum_{n=1}^{N-1} q_{N, n}\left(L_{N}(x)-L_{N-2}(x)\right) L_{n}(y) .
\end{gathered}
$$

With the convention $L_{-1}=0$, we choose $\mathbf{w}_{N}=\left(w_{N}, z_{N}\right)$ with

$$
\begin{gathered}
w_{N}(x, y)=-\sum_{m=0}^{N-1} \sum_{n=0}^{m} q_{m, n} \frac{L_{m+1}(x)-L_{m-1}(x)}{2 m+1} L_{n}(y) \\
-\sum_{m=0}^{N-1} q_{m, N} \frac{L_{m+1}(x)-L_{m-1}(x)}{2 m+1}\left(L_{N}(y)-L_{N-2}(y)\right)
\end{gathered}
$$

and

$$
\begin{array}{r}
z_{N}(x, y)=-\sum_{m=0}^{N-1} \sum_{n=m+1}^{N-1} q_{m, n} L_{m}(x) \frac{L_{n+1}(y)-L_{n-1}(y)}{2 n+1} \\
-\sum_{n=1}^{N-1} q_{N, n}\left(L_{N}(x)-L_{N-2}(x)\right) \frac{L_{n+1}(y)-L_{n-1}(y)}{2 n+1} .
\end{array}
$$

Then, in view of (2.8), we have

$$
\operatorname{div} \mathbf{w}_{N}=-q_{N} \quad \text { and } \quad \mathbf{w}_{N} \in X_{N},
$$

since $\mathbf{w}_{N} \cdot \mathbf{n}_{\mid \Gamma^{1}}=z_{N \mid \Gamma^{1}}=0$. As in [6, Chap. IV], we prove

$$
\begin{aligned}
\left\|q_{N}\right\|_{L^{2}(\Omega)}^{2} \geq c\left(\sum_{m=0}^{N-1} \sum_{n=0}^{N-1} q_{m, n}^{2}\right. & \frac{1}{\left(m+\frac{1}{2}\right)\left(n+\frac{1}{2}\right)} \\
& \left.+\frac{1}{N+\frac{1}{2}}\left(\sum_{m=0}^{N-1} q_{m, N}^{2} \frac{1}{m+\frac{1}{2}}+\sum_{n=1}^{N-1} q_{N, n}^{2} \frac{1}{n+\frac{1}{2}}\right)\right) .
\end{aligned}
$$


Hence, we derive, in the same way as in [8, Section 24]

$$
\left\|\frac{\partial w_{N}}{\partial x}\right\|_{L^{2}(\Omega)}+\left\|\frac{\partial z_{N}}{\partial y}\right\|_{L^{2}(\Omega)} \leq c\left\|q_{N}\right\|_{L^{2}(\Omega)} .
$$

Next, setting $w_{N}^{*}(x, y)=w_{N}(x, y)-q_{0,0} L_{1}(x)-q_{0, N} L_{1}(x)\left(L_{N}(y)-L_{N-2}(y)\right)$, we note that $w_{N}^{*}( \pm 1, y)=0$ for $-1 \leq y \leq 1$. Then, the Poincaré-Friedrichs inequality, applied with respect to $x$ or $y$, yields

$$
\left\|w_{N}^{*}\right\|_{L^{2}(\Omega)} \leq c\left\|\frac{\partial w_{N}^{*}}{\partial x}\right\|_{L^{2}(\Omega)} \quad \text { and } \quad\left\|z_{N}\right\|_{L^{2}(\Omega)} \leq c\left\|\frac{\partial z_{N}}{\partial y}\right\|_{L^{2}(\Omega)} .
$$

Hence, owing to (4.35) and the estimate

$$
\sqrt{q_{0,0}^{2}+\frac{q_{0, N}^{2}}{N+\frac{1}{2}}} \leq c\left\|q_{N}\right\|_{L^{2}(\Omega)},
$$

which is derived from (4.34), we obtain

$$
\left\|w_{N}\right\|_{L^{2}(\Omega)}+\left\|z_{N}\right\|_{L^{2}(\Omega)} \leq c\left\|q_{N}\right\|_{L^{2}(\Omega)} .
$$

Finally (4.35) and (4.36) imply

$$
\left\|\mathbf{w}_{N}\right\|_{H(\operatorname{div} ; \Omega)} \leq c\left\|q_{N}\right\|_{L^{2}(\Omega)}
$$

and, in view of (4.33), the choice $\mathbf{v}_{N}=\mathbf{w}_{N}$ in $b_{N}^{*}\left(\mathbf{v}_{N}, q_{N}\right)$ is available and gives the inf-sup condition (4.30).

From the previous lemma, we derive the following theorem.

Theorem 4.10 Let $\mathbf{f}$ be in $C^{0}(\bar{\Omega})^{2}$. Then problem (4.27), (4.28) has a unique solution $\left(\mathbf{u}_{N}, p_{N}\right)$ satisfying

$$
\left\|\mathbf{u}_{N}\right\|_{H(\operatorname{div} ; \Omega)}+\left\|p_{N}\right\|_{L^{2}(\Omega)} \leq C\left\|\mathcal{I}_{N} \mathbf{f}\right\|_{L^{2}(\Omega)^{2}} .
$$

Sketch of the proof. From(4.27), we derive $\left(\mathbf{u}_{N}, \mathbf{u}_{N}\right)_{N}=\left(\mathcal{I}_{N} \mathbf{f}, \mathbf{u}_{N}\right)_{N}$. Then, Owing to $\operatorname{div} \mathbf{u}_{N}=0$ and (2.13), we derive $\left\|\mathbf{u}_{N}\right\|_{H(\text { div } ; \Omega)} \leq 3\left\|\mathcal{I}_{N} \mathbf{f}\right\|_{L^{2}(\Omega)}$. Next, the inf-sup condition (4.30) and (4.27) imply

$$
\left\|p_{N}\right\|_{L^{2}(\Omega)} \leq \frac{1}{c} \sup _{\mathbf{v}_{N} \in X_{N}} \frac{b_{N}^{*}\left(\mathbf{v}_{N}, p_{N}\right)}{\left\|v_{N}\right\|_{H(\operatorname{div} ; \Omega)}} \leq \frac{1}{c} \sup _{\mathbf{v}_{N} \in X_{N}} \frac{\left(\mathcal{I}_{N} \mathbf{f}, \mathbf{v}_{N}\right)_{N}-\left(\mathbf{u}_{N}, \mathbf{v}_{N}\right)_{N}}{\left\|v_{N}\right\|_{H(\operatorname{div} ; \Omega)}} .
$$

Hence, in view of (2.13), we obtain

$$
\left\|p_{N}\right\|_{L^{2}(\Omega)} \leq \frac{18}{c}\left\|\mathcal{I}_{N} \mathbf{f}\right\|_{L^{2}(\Omega)},
$$

which ends the proof.

Next, in the same way as in Theorem 4.2, we prove an optimal error estimate.

Theorem 4.11 Assume that the solution $(\mathbf{u}, p)$ of problem (4.27), (4.28) belongs to $H^{s}(\Omega)^{2}$ $\times H^{s}(\Omega), s \geq 0$, and the data $\mathbf{f}$ belongs to $H^{\sigma}(\Omega)^{2}, \sigma>1$. Then, the following estimate holds

$$
\left\|\mathbf{u}-\mathbf{u}_{N}\right\|_{L^{2}(\Omega)^{2}}+\left\|p-p_{N}\right\|_{L^{2}(\Omega)} \leq c\left(N^{-s}\left(\|\mathbf{u}\|_{H^{s}(\Omega)^{2}}+\|p\|_{H^{s}(\Omega)}\right)+N^{-\sigma}\|\mathbf{f}\|_{H^{\sigma}(\Omega)^{2}}\right) .
$$

Sketch of the proof. Again, from the abstract error estimate for the approximation of saddle-point problems (see [7, Chap. IV]), we derive the following estimate: 


$$
\begin{array}{r}
\left\|\mathbf{u}-\mathbf{u}_{N}\right\|_{L^{2}(\Omega)^{2}}+\left\|p-p_{N}\right\|_{\left.L^{2} \Omega\right)} \leq c\left(\inf _{\mathbf{w}_{N} \in V_{N}}\left\|\mathbf{u}-\mathbf{w}_{N}\right\|_{L^{2}(\Omega)^{2}}\right. \\
+\inf _{\mathbf{v}_{N} \in X_{N}}\left(\left\|\mathbf{u}-\mathbf{v}_{N}\right\|_{L^{2}(\Omega)^{2}}+\sup _{\mathbf{z}_{N} \in X_{N}} \frac{\int_{\Omega} \mathbf{v}_{N}(\mathbf{x}) \cdot \mathbf{z}_{N}(\mathbf{x}) d \mathbf{x}-\left(\mathbf{v}_{N}, \mathbf{z}_{N}\right)_{N}}{\left\|\mathbf{z}_{N}\right\|_{L^{2}(\Omega)^{2}}}\right) \\
+\inf _{q_{N} \in M_{N}}\left(\left\|p-q_{N}\right\|_{L^{2}(\Omega)}+\sup _{\mathbf{z}_{N} \in X_{N}} \frac{b^{*}\left(\mathbf{z}_{N}, q_{N}\right)-b_{N}^{*}\left(\mathbf{z}_{N}, q_{N}\right)}{\left\|\mathbf{z}_{N}\right\|_{L^{2}(\Omega)^{2}}}\right) \\
\left.+\sup _{\mathbf{z}_{N} \in X_{N}} \frac{\int_{\Omega} \mathbf{f}(\mathbf{x}) \cdot \mathbf{z}_{N}(\mathbf{x}) d \mathbf{x}-\left(\mathbf{f}, \mathbf{z}_{N}\right)_{N}}{\left\|\mathbf{z}_{N}\right\|_{L^{2}(\Omega)^{2}}}\right),
\end{array}
$$

where $V_{N}$ is defined by

$$
V_{N}=\left\{\mathbf{w}_{N} \in X_{N} ; \forall q_{N} \in M_{N}, b_{N}^{*}\left(\mathbf{w}_{N}, q_{N}\right)=0\right\} .
$$

Moreover, we still have (see [11, CH. II, (1.16)])

$$
\inf _{\mathbf{w}_{N} \in V_{N}}\left\|\mathbf{u}-\mathbf{w}_{N}\right\|_{L^{2}(\Omega)^{2}} \leq \frac{c}{\gamma} \inf _{\mathbf{v}_{N} \in X_{N}}\left(\left\|\mathbf{u}-\mathbf{v}_{N}\right\|_{L^{2}(\Omega)^{2}}\right) .
$$

We end the proof in the same way as in Theorem 4.2.

Remark 4.12 As for the first discretization, problem (4.27, (4.28) can be interpreted as a collocation scheme. In the same way, we prove that (4.27), (4.28) is equivalent to the set of equations for $\mathbf{u}_{N}$ in $X_{N}$ and $p_{N}$ in $M_{N}$ :

$$
\begin{array}{rr}
\mathbf{u}_{N}(\mathbf{x})+\nabla p_{N}(\mathbf{x})=\mathbf{f}(\mathbf{x}), & \forall \mathbf{x} \in \Xi_{N} \cap \Omega, \\
\frac{2}{N(N+1)}\left(\left(\mathbf{u}_{N} \cdot \mathbf{n}\right)(\mathbf{x})+\frac{\partial p_{N}}{\partial n}(\mathbf{x})\right)=(\mathbf{f} \cdot \mathbf{n})(\mathbf{x}), & \forall \mathbf{x} \in \Xi_{N} \cap \Gamma^{2}, \\
\operatorname{div} \mathbf{u}_{N}(\mathbf{x})=0, & \forall \mathbf{x} \in \Xi_{N} .
\end{array}
$$

Therefore, the discrete solution $\mathbf{u}_{N}$ is exactly divergence-free, which is important for some applications.

\section{NUMERICAL RESULTS}

The convergence of the methods corresponding to the first and third discretizations were tested in a problem of the type (1.1)-(1.4), with homogeneous boundary conditions. Precisely, we tested the convergence of these methods to the exact solution $\mathbf{u}(x, y)=$ $\left(\pi x^{2} \cos (\pi y),-2 x \sin (\pi y)\right.$ and $p(x, y)=y \sin (\pi x)$, which means that we studied the convergence of these methods for Problem (1.1)-(1.4), with

$$
\mathbf{f}(x, y)=\left(\pi x^{2} \cos (\pi y)+\pi y \cos (\pi x),-2 x \sin (\pi y)+\sin (\pi x)\right)
$$

and homogeneous boundary conditions. In addition, we tested the convergence to 0 of the divergence for both methods.

We shall use the Lagrange polynomials. We denote $l_{r}$ the Lagrange polynomial associated to the Gauss-Lobatto point $\xi_{r}, 0 \leq r \leq N$, the expression of which is

$$
l_{r}(x)=\frac{\prod_{j=0, j \neq r}^{N}\left(x-\xi_{j}\right)}{\prod_{j=0, j \neq r}^{N}\left(\xi_{r}-\xi_{j}\right)} .
$$

The derivative $l_{r}^{\prime}$ verifies the following equalities 


$$
\begin{gathered}
\forall r=0, \ldots, N, \forall m=0, \ldots, N, r \neq m, l_{r}^{\prime}\left(\xi_{m}\right)=\frac{\prod_{j=0, j \neq m}^{N}\left(\xi_{m}-\xi_{j}\right)}{\prod_{j=0, j \neq r}^{N}\left(\xi_{r}-\xi_{j}\right)}\left(\frac{1}{\xi_{m}-\xi_{r}}\right), \\
\forall r=0, \ldots, N, l_{r}^{\prime}\left(\xi_{r}\right)=\sum_{j=0, j \neq r}^{N} \frac{1}{\xi_{r}-\xi_{j}} .
\end{gathered}
$$

\section{Uzawa's algorithm}

Problem (4.3), (4.4), Problem (4.13), (4.14) and Problem (4.27), (4.28) are equivalent to a linear system of the type :

$$
\left\{\begin{array}{l}
M U+D P=M F \\
D^{T} U=0
\end{array}\right.
$$

The unkowns are the vectors $U$ and $P$ which represent respectively the velocity and the pressure values on a given grid points. The data $f$ is representated by the vector $\mathrm{F}$ on the same grid points. The diagonal matrix $M$ is the weight matrix, while the matrix $D$ is associated to the form $b_{N}$ for the first and second spectral discretizations and to the form $b_{N}^{*}$ for the third spectral discretization and $D^{T}$ is the transposed matrix of $D$.

Uzawa's algorithm consists in rewriting the first equation of system (5.4) as: $U=F-M^{-1} D P$ and substituting in the second equation. We obtain a new equation for the pressure $P$ :

$$
\left(D^{T} M^{-1} D\right) P=D^{T} F .
$$

Next, we solve this symetric system either directly if the matrix $D^{T} M^{-1} D$ is invertible or by diagonalizing the matrix $D^{T} M^{-1} D$ if not, because the spurious modes correspond to eigenvalues of the matrix equal to zero. Next, we compute the velocity via the formula

$$
U=F-M^{-1} D P
$$

and its divergence by multiplying $U$ on the left by the matrix $D^{T}$. Finally, we test the convergence to 0 of its divergence.

\section{Implementation of the rst discretization}

We take $\left(l_{j}(x) l_{k}(y), 0\right),\left(0, l_{j}(x) l_{k}(y)\right), 0 \leq i, j \leq N$ as a basis of $X_{N}$ and $l_{r}(x) l_{s}(y), 1 \leq r \leq N-1,0 \leq s \leq N$ as a basis of $P_{N}(\Omega) \cap M$. The unknowns are the velocity $\mathbf{u}_{N}=\left(u_{N}^{1}, u_{N}^{2}\right)$ and the pressure $p_{N}$. For $j=1,2$, for $0 \leq r, s \leq N$, we denote $U_{r, s}^{j, N}=u_{N}^{j}\left(\xi_{r}, \xi_{s}\right), F_{r, s}^{j, N}=f_{j}\left(\xi_{r}, \xi_{s}\right)$, where $\mathbf{f}=\left(f_{1}, f_{2}\right)$ represents the data, and, for $1 \leq r \leq N-1$, for $0 \leq s \leq N$, we denote $P_{r, s}^{N}=p_{N}\left(\xi_{r}, \xi_{s}\right)$. So, we have

$$
u_{N}^{j}(x, y)=\sum_{r, s=0}^{N} U_{r, s}^{j, N} l_{r}(x) l_{s}(y), j=1,2 \text { and } p_{N}(x, y)=\sum_{r=1}^{N-1} \sum_{s=0}^{N} P_{r, s}^{N} l_{r}(x) l_{s}(y) .
$$

Let us define the $(N+1)^{2} \times(N+1)^{2}$ diagonal matrix $\tilde{M}=\left(m_{(j, k),(r, s)}\right)_{0 \leq j, k, r, s \leq N}$ with

$$
m_{(j, k),(r, s)}= \begin{cases}0 & \text { if }(r, s) \neq(j, k) \\ \rho_{j} \rho_{k} & \text { if }(r, s)=(j, k),\end{cases}
$$

and the $2(N+1)^{2} \times 2(N+1)^{2}$ diagonal matrix

$$
M=\left(\begin{array}{cc}
\tilde{M} & 0 \\
0 & \tilde{M}
\end{array}\right) .
$$

By setting $\mathbf{v}_{N}(x, y)=\left(l_{j}(x) l_{k}(y), 0\right)$, for $0 \leq j, k \leq N$ and, next, $\mathbf{v}_{N}(x, y)=\left(0, l_{j}(x) l_{k}(y)\right.$ in (4.3), (4.4), we obtain the matrix system (5.4) where the column matrices $U, P$ and $F$ are defined by 


$$
U=\left(\begin{array}{c}
U_{j, k}^{1, N} \\
U_{j, k}^{2, N}
\end{array}\right), P=\left(P_{r, s}^{N}\right) \text { and } F=\left(\begin{array}{c}
F_{j, k}^{1, N} \\
F_{j, k}^{2, N}
\end{array}\right), 0 \leq j, k, s \leq N, 1 \leq r \leq N-1,
$$

and, with $(., .)_{N}$ defined by $(4.1)$, the $2(N+1)^{2} \times\left(N^{2}-1\right)$ matrix $D$ by

$$
D=\left(\begin{array}{c}
D^{1, N} \\
D^{2, N}
\end{array}\right) \text { with } D^{1, N}=\left(\left(l_{j}(x) l_{k}(y), l_{r}^{\prime}(x) l_{s}(y)\right)_{N}\right), D^{2, N}=\left(\left(l_{j}(x) l_{k}(y), l_{r}(x) l_{s}^{\prime}(y)\right)_{N}\right),
$$

where $(j, k)$ represents the row index, $(r, s)$ the column index, $0 \leq i, j, s \leq N$, $1 \leq r \leq N-1$, for the matrices $D^{1, N}$ and $D^{2, N}$. Note that $U$ and $F$ are $2(N+1)^{2} \times 1$ matrices and $P$ is a $\left(N^{2}-1\right) \times 1$ matrix.

Proposition 5.1 The $\left(N^{2}-1\right) \times\left(N^{2}-1\right)$ square matrix $D^{T} M^{-1} D$ is invertible.

Proof. We just have to prove that the rank of the matrix $D$ is $N^{2}-1$. Let us assume that the rank of the matrix $D$ is strictly smaller than $N^{2}-1$. Then, there exist a sequence of real number $\left(q_{r, s}\right), 1 \leq r \leq N-1,0 \leq s \leq N$, where all the real numbers $q_{r, s}$ are not equal to zero, such that

$$
\sum_{r=1}^{N-1} \sum_{s=0}^{N} q_{r, s} D_{r, s}=0
$$

where $D_{r, s}$ are the column vectors of the matrix $D$. Setting $q=\sum_{r=1}^{N-1} \sum_{s=0}^{N} q_{r, s} l_{r}(x) l_{s}(y)$, the previous equality is equivalent to

$$
\forall \mathbf{v}_{N} \in X_{N}, b\left(\mathbf{v}_{N}, q\right)=0,
$$

which is in contradiction with the property that there is no spurious mode.

We have to compute the matrix $D^{T} M^{-1} D=\left(b_{(t, u),(r, s)}\right), 1 \leq r, t \leq N-1,0 \leq s, u \leq N$. Owing to the previous expression of the matrices $D$ and $M$, we obtain

$$
\begin{aligned}
b_{(t, u),(r, s)} & =\sum_{m, k=0}^{N} \frac{1}{\rho_{m} \rho_{k}}\left(l_{m}(x) l_{k}(y), l_{t}^{\prime}(x) l_{u}(y)\right)_{N}\left(l_{m}(x) l_{k}(y), l_{r}^{\prime}(x) l_{s}(y)\right)_{N} \\
& +\sum_{m, k=0}^{N} \frac{1}{\rho_{m} \rho_{k}}\left(l_{m}(x) l_{k}(y), l_{t}(x) l_{u}^{\prime}(y)\right)_{N}\left(l_{m}(x) l_{k}(y), l_{r}(x) l_{s}^{\prime}(y)\right)_{N} .
\end{aligned}
$$

Next, we change the numbering for the matrix $D^{T} M^{-1} D$. Let us define the mapping $\varphi$ by

$$
\forall(r, s), 1 \leq r \leq N-1,0 \leq s \leq N, \varphi(r, s)=1+(r-1)(N+1)+s .
$$

Note that $\varphi$ is a one to one mapping from $\{1, \ldots, N-1\} \times\{0, \ldots, N\}$ to $\left\{1, \ldots, N^{2}-1\right\}$ and we note

$$
\forall 1 \leq i \leq N^{2}-1, \varphi^{-1}(i)=\left(\psi_{1}(i), \psi_{2}(i)\right) .
$$

Note that $\psi_{1}(i)-1$ and $\psi_{2}(i)$ are respectively the quotient and the remainder of the euclidian division of $i-1$ by $N+1$. Then, we can denote

$$
D^{T} M^{-1} D=\left(a_{i, j}\right)_{1 \leq i, j \leq N^{2}-1} \text { with } a_{i, j}=b_{(t, u),(r, s)},
$$

where $(t, u)=\left(\psi_{1}(i), \psi_{2}(i)\right)$ and $(r, s)=\left(\psi_{1}(j), \psi_{2}(j)\right)$.

Computing the elements $a_{i, j}$ of the matrix $D^{T} M^{-1} D$ yields

1) if $\psi_{1}(i) \neq \psi_{1}(j)$ and $\psi_{2}(i) \neq \psi_{2}(j)$

$a_{i, j}=0$

$2)$ if $\psi_{1}(i) \neq \psi_{1}(j)$ and $\psi_{2}(i)=\psi_{2}(j)$

$a_{i, j}=\rho_{\psi_{2}(i)} \sum_{m=0}^{N} \rho_{m} l_{\psi_{1}(i)}^{\prime}\left(\xi_{m}\right) l_{\psi_{1}(j)}^{\prime}\left(\xi_{m}\right)$

3) if $\psi_{1}(i)=\psi_{1}(j)$ and $\psi_{2}(i) \neq \psi_{2}(j)$

$a_{i, j}=\rho_{\psi_{1}(i)} \sum_{m=0}^{N} \rho_{m} l_{\psi_{2}(i)}^{\prime}\left(\xi_{m}\right) l_{\psi_{2}(j)}^{\prime}\left(\xi_{m}\right)$ 
4) if $\psi_{1}(i)=\psi_{1}(j)$ and $\psi_{2}(i)=\psi_{2}(j)$, that is to say $\mathrm{i}=\mathrm{j}$ $a_{i, i}=\rho_{\psi_{2}(i)} \sum_{m=0}^{N} \rho_{m}\left(l_{\psi_{1}(i)}^{\prime}\right)^{2}+\rho_{\psi_{1}(i)} \sum_{m=0}^{N} \rho_{m}\left(l_{\psi_{2}(i)}^{\prime}\right)^{2}$.

Thus, most of elements of the matrix $D^{T} M^{-1} D$ are equal to zero.

Next, we determine the column matrix $D^{T} F=\left(c_{i, 1}\right)$ by

$$
\forall 1 \leq i \leq N^{2}-1, \quad c_{i, 1}=\rho_{\psi_{2}(i)} \sum_{m=0}^{N} \rho_{m} l_{\psi_{1}(i)}^{\prime}\left(\xi_{m}\right) F_{m, \psi_{2}(i)}^{1, N}+\rho_{\psi_{1}(i)} \sum_{m=0}^{N} \rho_{m} l_{\psi_{2}(i)}^{\prime}\left(\xi_{m}\right) F_{\psi_{1}(i), m}^{2, N} .
$$

Hence, owing to (5.2) and (5.3), we compute the list $\left[l_{k}^{\prime}\left(\xi_{m}\right), 0 \leq k, m \leq N\right]$, which allows us to determine the elements of the matrices $D^{T} M^{-1} D$ and $D^{T} F$. Since the matrix $D^{T} M^{-1} D$ is invertible, the equation $\left(D^{T} M^{-1} D\right) X=D^{T} F$ has a unique solution $X$. Then we derive easily the column matrix $P=\left(P_{r, s}^{N}\right)$ such that $P_{r, s}^{N}=X \varphi(r, s), 1 \leq r \leq N-1$, $0 \leq s \leq N$ and, next, the column matrix $\mathrm{U}$, thanks to the relation $U=F-M^{-1} D P$. Setting $P_{0, t}^{N}=P_{N, t}^{N}=0$ for $0 \leq t \leq N$ in accordance with the boundary conditions, we obtain

$$
U_{m, k}^{1, N}=F_{m, k}^{1, N}-\sum_{u=1}^{N-1} l_{u}^{\prime}\left(\xi_{m}\right) P_{u, k}^{N}, U_{m, k}^{2, N}=F_{m, k}^{2, N}-\sum_{t=0}^{N} l_{t}^{\prime}\left(\xi_{k}\right) P_{m, t}^{N} .
$$

Finally, we derive

$$
\begin{gathered}
\left(\operatorname{div} \mathbf{u}_{N}, \operatorname{div} \mathbf{u}_{N}\right)_{N}=\sum_{i, j=0}^{N} \rho_{i} \rho_{j}\left(\sum_{m=0}^{N}\left(U_{m, j}^{1, N} l_{m}^{\prime}\left(\xi_{i}\right)+U_{i, m}^{2, N} l_{m}^{\prime}\left(\xi_{j}\right)\right)\right)^{2}, \\
\left.\left(\mathbf{u}-\mathbf{u}_{N}, \mathbf{u}-\mathbf{u}_{N}\right)_{N}=\sum_{i, j=0}^{N} \rho_{i} \rho_{j}\left(u_{1}\left(\xi_{i}, \xi_{j}\right)-U_{i, j}^{1, N}\right)^{2}+\left(u_{2}\left(\xi_{i}, \xi_{j}\right)-U_{i, j}^{2, N}\right)^{2}\right), \\
\left(p-p_{N}, p-p_{N}\right)_{N}=\sum_{i=1}^{N-1} \sum_{j=0}^{N} \rho_{i} \rho_{j}\left(p\left(\xi_{i}, \xi_{j}\right)-P_{i, j}^{N}\right)^{2} .
\end{gathered}
$$

We give the values of $\left(\operatorname{div} \mathbf{u}_{N}, \operatorname{div} \mathbf{u}_{N}\right)_{N}^{\frac{1}{2}},\left(p-p_{N}, p-p_{N}\right)_{N}^{\frac{1}{2}}$ and $\left(\mathbf{u}-\mathbf{u}_{N}, \mathbf{u}-\mathbf{u}_{N}\right)_{N}^{\frac{1}{2}}$ for $N$ between 4 and 21 .

\begin{tabular}{ccccccc}
$\mathbf{N}$ & 4 & \multicolumn{1}{c}{5} & \multicolumn{1}{c}{6} & \multicolumn{1}{c}{7} & \multicolumn{1}{c}{8} & \multicolumn{1}{c}{$\mathbf{c}$} \\
$\left(\operatorname{div} \mathbf{u}_{N}, \operatorname{div} \mathbf{u}_{N}\right)_{N}^{\frac{1}{2}}$ & 6,85 & 0,27 & 1,12 & 0,0155 & 0,080 & $5,16.10^{-4}$ \\
$\left(p-p_{N}, p-p_{N}\right)_{N}^{\frac{1}{2}}$ & 0,06 & 0,02 & $2,7.10^{-3}$ & $7,75.10^{-4}$ & $7,59.10^{-5}$ & $1,74.10^{-5}$ \\
$\left(\mathbf{u}-\mathbf{u}_{N}, \mathbf{u}-\mathbf{u}_{N}\right)_{N}^{\frac{1}{2}}$ & 1,07 & 0,045 & 0,010 & $1,56.10^{-3}$ & $4,65.10^{-3}$ & $3,52.10^{-5}$
\end{tabular}

$\begin{array}{ccccccc}\mathbf{N} & 10 & 11 & 12 & 13 & 14 & 15 \\ \left(\operatorname{div} \mathbf{u}_{N}, \operatorname{div} \mathbf{u}_{N}\right)_{N}^{\frac{1}{2}} & 3,14.10^{-3} & 1,11.10^{-5} & 7,86.10^{-5} & 1,70.10^{-7} & 1,36.10^{-6} & 1,92.10^{-9} \\ \left(p-p_{N}, p-p_{N}\right)_{N}^{\frac{1}{2}} & 1,44.10^{-6} & 2,78.10^{-7} & 1,96.10^{-8} & 3,27.10^{-9} & 2,03.10^{-10} & 2,97.10^{-11} \\ \left(\mathbf{u}-\mathbf{u}_{N}, \mathbf{u}-\mathbf{u}_{N}\right)_{N}^{\frac{1}{2}} & 1,33.10^{-4} & 5,6.10^{-7} & 2,56.10^{-6} & 6,62.10^{-9} & 3,54.10^{-8} & 6,03.10^{-11}\end{array}$

$\begin{array}{ccccccc}\mathbf{N} & 16 & 17 & 18 & 19 & 20 & 21 \\ \left(\operatorname{div} \mathbf{u}_{N}, \operatorname{div} \mathbf{u}_{N}\right)_{N}^{\frac{1}{2}} & 1,73.10^{-8} & 1,68.10^{-11} & 1,69.10^{-10} & 1,02.10^{-12} & 3,79.10^{-12} & 1,91.10^{-11} \\ \left(p-p_{N}, p-p_{N}\right)_{N}^{\frac{1}{2}} & 1,64.10^{-12} & 2,15.10^{-13} & 1,06.10^{-14} & 5,50.10^{-15} & 1,09.10^{-14} & 1,06.10^{-14} \\ \left(\mathbf{u}-\mathbf{u}_{N}, \mathbf{u}-\mathbf{u}_{N}\right)_{N}^{\frac{1}{2}} & 3,70.10^{-10} & 4,37.10^{-13} & 3,03.10^{-12} & 3,03.10^{-14} & 9,03.10^{-14} & 2,69.10^{-13}\end{array}$

\section{Table 4.1}

We shall comment these results on comparing them with these ones of the third discretization. 


\section{Implementation of the first discretization}

We only sketch the method because the first and third discretizations are rather similar, but we shall point out the differences between both discretizations.

First, the space $X_{N}$ is not the same, because boundary conditions are taken in account. Thus, we take $\left(l_{j}(x) l_{k}(y), 0\right),\left(0, l_{s}(x) l_{r}(y)\right), 0 \leq i, j, s \leq N, 1 \leq r \leq N-1$ as a basis of $X_{N}$.

Second, we shall compute the pressure $p_{N}$ as the orthogonal projection on $M_{N}$ of an element of $\mathbb{P}_{N}(\Omega)$ and we take $l_{r}(x) l_{s}(y), 0 \leq r, s \leq N$ as a basis of $\mathbb{P}_{N}(\Omega)$. For $0 \leq r, s \leq$ $N$, we denote $U_{r, s}^{1, N}=u_{N}^{1}\left(\xi_{r}, \xi_{s}\right), P_{r, s}^{N}=p_{N}\left(\xi_{r}, \xi_{s}\right)$ and for $0 \leq r \leq N, 1 \leq s \leq N-1$, we denote $U_{r, s}^{2, N}=u_{N}^{2}\left(\xi_{r}, \xi_{s}\right)$. Let us define the matrix $\tilde{M}_{1}=\tilde{M}$, where $\tilde{M}$ is given by (5.7), the $\left(N^{2}-1\right) \times\left(N^{2}-1\right)$ matrix $\tilde{M}_{2}=\left(m_{(j, k),(r, s)}\right)$ with

$$
m_{(j, k),(r, s)}=\left\{\begin{array}{ll}
0 & \text { if }(r, s) \neq(j, k) \\
\rho_{j} \rho_{k} & \text { if }(r, s)=(j, k),
\end{array} \quad 0 \leq j, r \leq N, 1 \leq k, s \leq N-1\right.
$$

and the $2 N(N+1) \times 2 N(N+1)$ diagonal matrix

$$
M_{*}=\left(\begin{array}{cc}
\tilde{M}_{1} & 0 \\
0 & \tilde{M}_{2}
\end{array}\right)
$$

In the same way as the first discretization, we derive the following matrix system

$$
\left\{\begin{array}{l}
M_{*} U+D_{*} P=M_{*} F, \\
D_{*}^{T} U=0
\end{array},\right.
$$

where the column matrices $U, P$ and $F$ are defined by

$$
U=\left(\begin{array}{c}
U_{j, k}^{1, N} \\
U_{t, u}^{2, N}
\end{array}\right), P=\left(P_{r, s}^{N}\right) \text { and } F=\left(\begin{array}{c}
F_{j, k}^{1, N} \\
F_{t, u}^{2, N}
\end{array}\right), 0 \leq j, k, r, s, t \leq N, 1 \leq u \leq N-1
$$

and the $2 N(N+1) \times(N+1)^{2}$ matrix $D_{*}$ by

$D_{*}=\left(\begin{array}{c}D_{*}^{1, N} \\ D_{*}^{2, N}\end{array}\right)$ with $D_{*}^{1, N}=\left(-\left(l_{j}^{\prime}(x) l_{k}(y), l_{r}(x) l_{s}(y)\right)_{N}\right), D_{*}^{2, N}=\left(-\left(l_{t}(x) l_{u}^{\prime}(y), l_{r}(x) l_{s}(y)\right)_{N}\right)$.

We have to compute the matrix $D_{*}^{T} M_{*}^{-1} D_{*}=\left(b_{(t, u),(r, s)}^{*}\right), 0 \leq r, s, t, u \leq N$. In the same way as the first discretization, we obtain

$$
\begin{array}{r}
b_{(t, u),(r, s)}^{*}=\sum_{m, k=0}^{N} \frac{1}{\rho_{m} \rho_{k}}\left(l_{m}^{\prime}(x) l_{k}(y), l_{t}(x) l_{u}(y)\right)_{N}\left(l_{m}^{\prime}(x) l_{k}(y), l_{r}(x) l_{s}(y)\right)_{N} \\
+\sum_{m=0}^{N} \sum_{k=1}^{N-1} \frac{1}{\rho_{m} \rho_{k}}\left(l_{m}(x) l_{k}^{\prime}(y), l_{t}(x) l_{u}(y)\right)_{N}\left(l_{m}(x) l_{k}^{\prime}(y), l_{r}(x) l_{s}(y)\right)_{N} .
\end{array}
$$

Next, as in the first discretization, we change the numbering for the matrix $D_{*}^{T} M_{*}^{-1} D_{*}$. Let us define the mapping $\varphi_{*}$ by

$$
\forall(r, s), 0 \leq r, s \leq N, \boldsymbol{\varphi}_{*}(r, s)=r(N+1)+s+1 .
$$

Note that $\varphi_{*}$ is a one to one mapping from $\{0, \ldots, N\}^{2}$ to $\left\{1, \ldots,(N+1)^{2}\right\}$ and we note

$$
\forall 1 \leq i \leq N^{2}-1, \varphi_{*}^{-1}(i)=\left(\psi_{1}^{*}(i), \psi_{2}^{*}(i)\right) .
$$

Note that $\psi_{1}^{*}(i)$ and $\psi_{2}^{*}(i)$ are respectively the quotient and the remainder of the euclidian division of $i-1$ by $N+1$. Then, we can denote

$$
D_{*}^{T} M_{*}^{-1} D_{*}=\left(a_{i, j}^{*}\right)_{1 \leq i, j \leq(N+1)^{2}} \text { with } a_{i, j}^{*}=b_{(t, u),(r, s)}^{*},
$$


where $(t, u)=\left(\psi_{1}^{*}(i), \psi_{2}^{*}(i)\right)$ and $(r, s)=\left(\psi_{1}^{*}(j), \psi_{2}^{*}(j)\right)$.

Computing the elements $a_{i, j}^{*}$ of the matrix $D_{*}^{T} M_{*}^{-1} D_{*}$ yields

1) if $\psi_{1}^{*}(i) \neq \psi_{1}^{*}(j)$ and $\psi_{2}^{*}(i) \neq \psi_{2}^{*}(j)$

$a_{i, j}^{*}=0$

2) if $\psi_{1}^{*}(i) \neq \psi_{1}^{*}(j)$ and $\psi_{2}^{*}(i)=\psi_{2}^{*}(j)$

$a_{i, j}^{*}=\rho_{\psi_{1}^{*}(i)} \rho_{\psi_{1}^{*}(j)} \rho_{\psi_{2}^{*}(i)} \sum_{m=0}^{N} \frac{1}{\rho_{m}} l_{m}^{\prime}\left(\xi_{\psi_{1}^{*}(i)}\right) l_{m}^{\prime}\left(\xi_{\psi_{1}^{*}(j)}\right)$

3) if $\psi_{1}^{*}(i)=\psi_{1}^{*}(j)$ and $\psi_{2}^{*}(i) \neq \psi_{2}^{*}(j)$

$a_{i, j}^{*}=\rho_{\psi_{2}^{*}(i)} \rho_{\psi_{2}^{*}(j)} \rho_{\psi_{1}^{*}(i)} \sum_{m=1}^{N-1} \frac{1}{\rho_{m}} l_{m}^{\prime}\left(\xi_{\psi_{2}^{*}(i)}\right) l_{m}^{\prime}\left(\xi_{\psi_{2}^{*}(j)}\right)$

4) if $\psi_{1}^{*}(i)=\psi_{1}^{*}(j)$ and $\psi_{2}^{*}(i)=\psi_{2}^{*}(j)$, that is to say $\mathrm{i}=\mathrm{j}$

$\left.\left.a_{i, i}^{*}=\left(\rho_{\psi_{1}^{*}(i)}\right)^{2} \rho_{\psi_{2}^{*}(i)}\right) \sum_{m=0}^{N} \frac{1}{\rho_{m}}\left(l_{m}^{\prime}\left(\xi_{\psi_{1}^{*}(i)}\right)\right)^{2}+\left(\rho_{\psi_{2}^{*}(i)}\right)^{2} \rho_{\psi_{1}^{*}(i)}\right) \sum_{m=1}^{N-1} \frac{1}{\rho_{m}}\left(l_{m}^{\prime}\left(\xi_{\psi_{2}^{*}(i)}\right)\right)^{2}$. zero.

Next, we determine the column matrix $D_{*}^{T} F=\left(c_{i, 1}^{*}\right)$ by

$$
\forall 1 \leq i \leq(N+1)^{2}, c_{i, 1}=-\rho_{\psi_{1}(i)} \rho_{\psi_{2}(i)}\left(\sum_{m=0}^{N} l_{m}^{\prime}\left(\xi_{\psi_{1}^{*}(i)}\right) F_{m, \psi_{2}(i)}^{1, N}+\sum_{m=1}^{N-1} l_{k}^{\prime}\left(\xi_{\psi_{2}^{*}(i)}\right) F_{\psi_{1}(i), m}^{2, N}\right) .
$$

Now, we deal with the main difference between both discretizations. In the third discretization, the matrix $D_{*}^{T} M_{*}^{-1} D_{*}$ is not invertible and the computation of the pressure is more complicated. First, let $q_{N}=\sum_{t, u=0}^{N} q_{t, u} l_{t}(x) l_{u}(y)$ be a spurious mode, that is to say an element of the space $\mathcal{Z}_{N}^{*}$ defined in Lemma 4.8. In the same way as in the proof of Proposition 5.1, considering that the matrices $D_{*}^{T} M_{*}^{-1} D_{*}$ and $D_{*}$ have the same rank, we obtain the following equivalences

$$
q_{N} \in \mathcal{Z}_{N}^{*} \Longleftrightarrow D_{*} Q=0 \Longleftrightarrow\left(D_{*}^{T} M_{*}^{-1} D_{*}\right) Q=0,
$$

where $Q$ is the column vector $\left(q_{t, u}\right)_{0 \leq t, u \leq N}$. We can consider the matrix $D_{*}^{T} M_{*}^{-1} D_{*}$ as the matrix of a linear mapping $f$ from the vector space $\mathbb{P}_{N}(\Omega)$ into itself equipped with the basis $\mathcal{B}=\left(l_{i}(x) l_{j}(y)\right)_{0 \leq i, j \leq N}$. Therefore, $\mathcal{Z}_{N}^{*}$ is the eigenspace associated to the eigenvalue equal to 0 of the linear mapping $f$ or, equivalently, of its matrix $D_{*}^{T} M_{*}^{-1} D_{*}$. Note that this basis is orthonormal for the scalar product $(., .)_{N}$. We can diagonalize the positive symmetric matrix $D_{*}^{T} M_{*}^{-1} D_{*}$ and, thus, there exist a diagonal matrix $\Lambda$ and an orthogonal matrix $R$ such that $D_{*}^{T} M_{*}^{-1} D_{*}=R \Lambda R^{-1}$ and such that the diagonal elements of $\Lambda$, that is to say $\left(\lambda_{i, i}\right)_{1 \leq i \leq(N+1)^{2}}$ are in increasing order. Note that the matrix $\Lambda$ is the matrix of $f$ in a basis $\mathcal{B}^{\prime}=\left(h_{i}\right)_{1 \leq i \leq(N+1)^{2}}$ of $\mathbb{P}_{N}(\Omega)$, which is orthonormal for the scalar product $(., .)_{N}$, the elements of which are the eigenvectors of the matrix $D_{*}^{T} M_{*}^{-1} D_{*}$. Let $P^{\prime}$ be the column matrix the elements of which are the components of the pressure $p_{N}$ in the new basis $\mathcal{B}^{\prime}$ of $\mathbb{P}_{N}(\Omega)$. We have $P^{\prime}=R^{T} P=R^{-1} P$ and the following equivalence

$$
\left(D_{*}^{T} M_{*}^{-1} D\right) P=D_{*}^{T} F \Longleftrightarrow \Lambda P^{\prime}=R^{T} D_{*}^{T} F .
$$

Since $\mathcal{Z}_{N}^{*}$ is a two dimensions space, $\mathcal{B}_{0}^{\prime}=\left(h_{1}, h_{2}\right)$ is the basis of $\mathcal{Z}_{N}^{*}$ and $\left(h_{i}\right)_{3 \leq i \leq(N+1)^{2}}$ is a basis of $M_{N}=\left(\mathcal{Z}_{N}^{*}\right)^{\perp}$. Therefore, we determine the column vectors $P^{\prime}$ and $P$ by

$$
P_{1}^{\prime}=P_{2}^{\prime}=0, P_{i}^{\prime}=\frac{1}{\lambda_{i, i}}\left(R^{T} D_{*}^{T} F\right)_{i}, 3 \leq i \leq(N+1)^{2} \text { and } P=R P^{\prime},
$$

and we have $P_{r, s}^{N}=P \boldsymbol{\varphi}_{*}(r, s), 0 \leq r, s \leq N$. In the same way as for the first discretization, we derive the column matrix $U$ by the relation $U=F-M_{*}^{-1} D_{*} P$, which gives, for $0 \leq j, k, t \leq N, 1 \leq u \leq N-1$,

$$
U_{j, k}^{1, N}=F_{j, k}^{1, N}+\frac{1}{\rho_{j}} \sum_{r=0}^{N} \rho_{r} l_{j}^{\prime}\left(\xi_{r}\right) P_{r, k}^{N}, U_{t, u}^{2, N}=F_{t, u}^{2, N}+\frac{1}{\rho_{u}} \sum_{r=0}^{N} \rho_{r} l_{u}^{\prime}\left(\xi_{r}\right) P_{t, r}^{N}
$$


Finally, considering the boundary conditions, we set $U_{t, 0}^{2, N}=U_{t, N}^{2, N}=0,0 \leq t \leq N$, and we obtain the same formulas as in the first discretization for $\left(\operatorname{div} \mathbf{u}_{N}, \operatorname{div} \mathbf{u}_{N}\right)_{N}$, $\left(\mathbf{u}-\mathbf{u}_{N}, \mathbf{u}-\mathbf{u}_{N}\right)_{N}$ and $\left(p-p_{N}, p-p_{N}\right)_{N}$. We also give the values of $\left(\operatorname{div} \mathbf{u}_{N}, \operatorname{div} \mathbf{u}_{N}\right)_{N}^{\frac{1}{2}}$, $\left(p-p_{N}, p-p_{N}\right)_{N}^{\frac{1}{2}}$ and $\left(\mathbf{u}-\mathbf{u}_{N}, \mathbf{u}-\mathbf{u}_{N}\right)_{N}^{\frac{1}{2}}$ for $N$ between 4 and 21 .

\begin{tabular}{cllcllcc}
$\mathbf{N}$ & \multicolumn{1}{c}{$\mathbf{4}$} & \multicolumn{1}{c}{$\mathbf{5}$} & \multicolumn{1}{c}{$\mathbf{6}$} & \multicolumn{1}{c}{$\mathbf{7}$} & $\mathbf{8}$ & $\mathbf{9}$ \\
$\left(\operatorname{div} \mathbf{u}_{N}, \operatorname{div} \mathbf{u}_{N}\right)_{N}^{\frac{1}{2}}$ & $1,84.10^{-16}$ & $5,45 \cdot 10^{-15}$ & $3,60 \cdot 10^{-16}$ & $3,05.10^{-15}$ & $8,76 \cdot 10^{-16}$ & $1,94 \cdot 10^{-14}$ \\
$\left(p-p_{N}, p-p_{N}\right)_{N}^{\frac{1}{2}}$ & 0,246 & 0,016 & 0,019 & 0,0039 & $8,43.10^{-4}$ & $1,55.10^{-3}$ \\
$\left(\mathbf{u}-\mathbf{u}_{N}, \mathbf{u}-\mathbf{u}_{N}\right)_{N}^{\frac{1}{2}}$ & 0,62 & 0,043 & 0,054 & $1,54.10^{-3}$ & $2,48.10^{-3}$ & $3,54.10^{-5}$
\end{tabular}

\begin{tabular}{cccccccc}
$\mathbf{N}$ & $\mathbf{1 0}$ & \multicolumn{1}{c}{$\mathbf{1 1}$} & \multicolumn{1}{c}{$\mathbf{1 2}$} & \multicolumn{1}{c}{$\mathbf{1 3}$} & \multicolumn{1}{c}{$\mathbf{1 4}$} & \multicolumn{1}{c}{$\mathbf{1 5}$} \\
$\left(\operatorname{div} \mathbf{u}_{N}, \operatorname{div} \mathbf{u}_{N}\right)_{N}^{\frac{1}{2}}$ & $1,24.10^{-14}$ & $9,28.10^{-15}$ & $2,7.10^{-13}$ & $9,68.10^{-13}$ & $4,25.10^{-13}$ & $3,43.10^{-13}$ \\
$\left(p-p_{N}, p-p_{N}\right)_{N}^{\frac{1}{2}}$ & $2,3.10^{-5}$ & $8,25.10^{-4}$ & $4,33.10^{-7}$ & $4,8.10^{-4}$ & $5,9.10^{-9}$ & $3,1.10^{-4}$ \\
$\left(\mathbf{u}-\mathbf{u}_{N}, \mathbf{u}-\mathbf{u}_{N}\right)_{N}^{\frac{1}{2}}$ & $7,0.10^{-5}$ & $5,7.10^{-7}$ & $1,34.10^{-6}$ & $6,79.10^{-9}$ & $1,85.10^{-8}$ & $6,23.10^{-11}$
\end{tabular}

$\begin{array}{llllllll}\mathbf{N} & 16 & 17 & 18 & 19 & 20 & 21\end{array}$

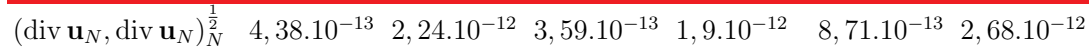

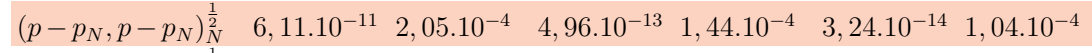
$\left(\mathbf{u}-\mathbf{u}_{N}, \mathbf{u}-\mathbf{u}_{N}\right)_{N}^{\frac{1}{2}} \quad 1,93.10^{-10} \quad 4,53.10^{-13} \quad 1,57.10^{-12} 3,66.10^{-14} \quad 1,12.10^{-13} \quad 2,38.10^{-13}$

Table 4.2

Logarithm to the basis 10 of $\left(\operatorname{div} \mathbf{u}_{N}, \operatorname{div} \mathbf{u}_{N}\right)_{N}^{\frac{1}{2}}$ in function of $N$ for the first and third discretizations

First discretization: Third discretization:

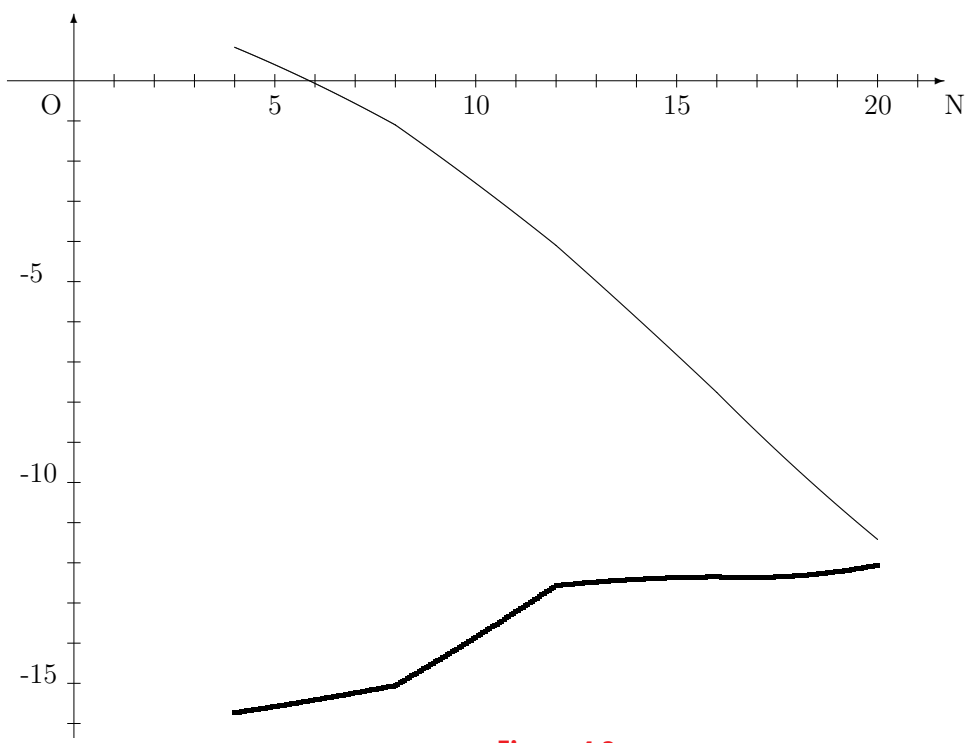

Figure 4.3 


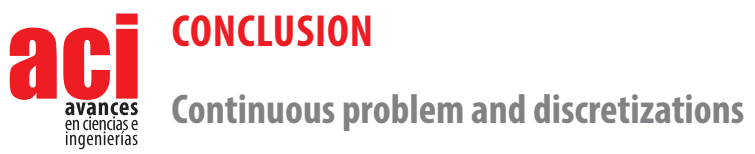

We studied the Darcy problem by defining two equivalent variational formulations corresponding to two different bilinear forms $b$ and $b^{*}$. The first one requests a more regular pressure, which is bounded in $H^{1}(\Omega)$. The second one is less classic and was introduced by A. Quarteroni and A. Valli (see [14]). This second variational formulation is important because it allowed us to constuct a spectral method which gives a divergence-free discrete solution.

Moreover, by studying a mixed problem of Dirichlet-Neumann for the Laplace operator, we proved regularity results with the solution $(\mathbf{u}, p)$ in $H^{1}(\Omega)^{2} \times H^{2}(\Omega)$ as long as the data is regular enough.

The first variational formulation led us to two spectral discretizations. In the first one, the discrete solution is not divergence-free, which is a disadvantage for some applications. However, we obtain a fully optimal error estimate for the velocity and the pressure. In the second discretization, it does not appear spurious modes for the pressure. Moreover, because of the exactness of the quadrature formulas, the error estimate is easier to obtain. However, the error estimate for the pressure is not optimal, because the inf-sup constant depends on $N$.

The second variational formulation led to a third spectral discretization. There are spurious modes, which complicate the study, but the discrete velocity is divergence-free, which is important when the system is a stage of solving of a time-dependent problem, and the error estimate for the velocity and the pressure is fully optimal. In conclusion, this third discretization is the best discretization and we will only use it hereafter.

\section{Comparison of both spectral discretizations}

Tables 4.1 and 4.2 test the convergence of respectively the first and third discretizations to the exact solutions. For the first discretization, we see the convergence to zero of $\left(\operatorname{div} \mathbf{u}_{N}, \operatorname{div} \mathbf{u}_{N}\right)_{N}^{\frac{1}{2}}$, about $10^{-1}$ for $N=8$ and about $10^{-12}$ for $N=19$. Concerning the third discretization, we see that the quantity $\left(\operatorname{div} \mathbf{u}_{N}, \operatorname{div} \mathbf{u}_{N}\right)_{N}^{\frac{1}{2}}$ is very small for all values of $N$, about $10^{-16}$ for $N=4$ and about $10^{-12}$ for $N=20$. Thus, we verify that, in the third discretization, the discrete solution $\mathbf{u}_{N}$ is exactly divergence-free, which is important, as we saw previously. This property appears clearly in Figure 4.3, which represents the logarithm to the basis 10 of the quantity $\left(\operatorname{div} \mathbf{u}_{N}, \operatorname{div} \mathbf{u}_{N}\right)_{N}^{\frac{1}{2}}$ as a function of $N$, for even $\mathrm{N}$ from 4 to 20 , for both discretizations.

Regarding the velocity, the discrete solution $\mathbf{u}_{N}$ converges fast to the exact solution $\mathbf{u}$ for both discretizations. For the pressure, we also obtain fast convergence, except for odd $N$ in the third discretization. 


\section{References}

[1] Achdou, Y., Bernardi, C. \& Coquel, F. "A priori a posteriori analysis of finite volume discretizations of Darcy's equations", Numer. Math. 96 (2003), 17-42.

[2] Azaïez, M., Bernardi, C. \& Grundmann, M. "Méthodes spectrales pour les équations du milieu poreux", East West Journal of Numerical Analysis, №. 2, 1994.

[3] Babuška, I. "The finite element method with lagrangian multipliers", Numer. Math. 20, pp. 179-192 (1973).

[4] Bègue, C., Conca, C., Murat, F. \& Pironneau, 0. "Les équations de Stokes et de Navier-Stokes avec des conditions aux limites sur la pression", Nonlinear Partial Differen- tial Equations and their Applications, Coll'ege de France Seminar, vol. IX (1988).

[5] Bernard, J.M. "Spectral discretizations of the Stokes equations with non standard boundary conditions", Journal of Scientific Computing, 20(3), 355-377 (2004).

[6] Bernardi, C., Canuto, C. \& Maday, Y. "Spectral approximations of the Stokes equa- tions with boundary conditions on the pressure", SIAM J. Numer. Anal. 28, № 2 (1991), p. 333-362.

[7] Bernardi, C. \& Maday, Y. Approximations spectrales de problèmes aux limites ellip-tiques, Springer-Verlag, Paris, 1992.

[8] Bernardi, C. \& Maday, Y. Spectral Methods, Handbook of Numerical Analysis, Vol. V, Paris (1997).

[9] Brezzi, F. "On the existence, uniqueness and approximation of saddle-point problems arising from Lagrange multipliers", R.A.I.R.0., Anal. Mumer. R2, PP.129-151 (1974).

[10] Chorin, A.J. " Numerical solution of the Navier-Stokes equations", Math. Comput. 22 (1968), 745-762.

[11] Girault, V. \& Raviart, P. A. Finite Element Methods for Navier-Stokes Equations,Springer-Verlag, Berlin (1986).

[12] Grisvard, P."Elliptic Problems in Nonsmooth Domains", Pitman Monographs and Studies in Mathematics, 24, Boston (1985).

[13] Nečas, J. les Méthodes Directes en Théorie des Equations Elliptiques, Masson, Paris (1967).

[14] Quarteroni, A.\& Valli, A. Domain decomposition methods for partial differential equations, Oxford science publication (1999).

[15] Temam, R. "Une méthode d'approximation de la solution des équations de Navier-Stokes", Bull. Soc. Math. France 98 (1968), 115-152. 